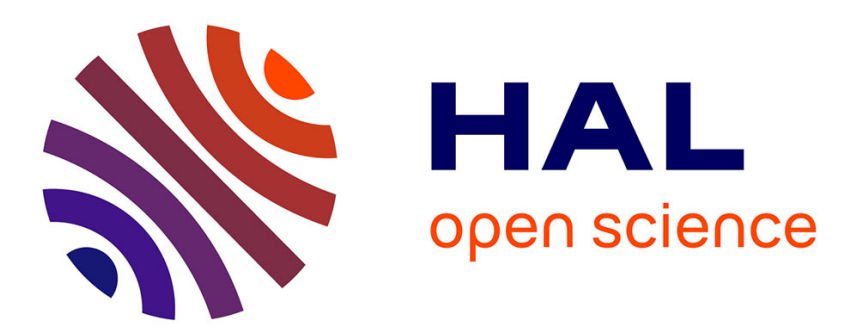

\title{
Smoothed Particle Hydrodynamics: A consistent model for interfacial multiphase fluid flow simulations
}

\author{
Abdelkader Krimi, Mehdi Rezoug, Sofiane Khelladi, Xesús Nogueira, Michael
}

Deligant, Luis Ramírez

\section{- To cite this version:}

Abdelkader Krimi, Mehdi Rezoug, Sofiane Khelladi, Xesús Nogueira, Michael Deligant, et al.. Smoothed Particle Hydrodynamics: A consistent model for interfacial multiphase fluid flow simulations. Journal of Computational Physics, 2018, 358, pp.53-87. 10.1016/j.jcp.2017.12.006 . hal02438236

\section{HAL Id: hal-02438236 \\ https://hal.science/hal-02438236}

Submitted on 14 Jan 2020

HAL is a multi-disciplinary open access archive for the deposit and dissemination of scientific research documents, whether they are published or not. The documents may come from teaching and research institutions in France or abroad, or from public or private research centers.
L'archive ouverte pluridisciplinaire HAL, est destinée au dépôt et à la diffusion de documents scientifiques de niveau recherche, publiés ou non, émanant des établissements d'enseignement et de recherche français ou étrangers, des laboratoires publics ou privés. 


\title{
Smoothed Particle Hydrodynamics : A consistent model for interfacial multiphase fluid flow simulations
}

\author{
Abdelkader Krimi ${ }^{\mathrm{a}, \mathrm{b}}$, Mehdi Rezoug ${ }^{\mathrm{a}}$, Sofiane Khelladi ${ }^{\mathrm{b}}$, Xesús Nogueira ${ }^{\mathrm{c}}$, \\ Michael Deligant ${ }^{\mathrm{b}}$, Luis Ramírez ${ }^{\mathrm{c}}$ \\ ${ }^{a}$ Institut de Recherche en Constructibilité, Ecole Spéciale des Travaux Publics, 28 avenue \\ du Président Wilson, 94230 Cachan, France \\ ${ }^{b}$ DynFluid Laboratory, Arts et Métiers ParisTech, 151 Boulevard de l'Hôpital, 75013 \\ Paris, France \\ ${ }^{c}$ Group of Numerical Methods in Engineering, Universidade da Coruña. Campus de \\ Elviña, 15071, A Coruña, Spain
}

\begin{abstract}
In this work, a consistent Smoothed Particle Hydrodynamics (SPH) model is proposed to deal with interfacial multiphase fluid flows simulation. A Continuum Stress Surface formulation (CSS) [1] was developed in the framework of SPH method using a non-conservative first order consistency operator to calculate the divergence of stress surface tensor. This enables the enhancement of the stability near the fluid interface. This formulation benefits of all the advantages of the one proposed by Adami et al [2] and, in addition, it can be applied to more than two phases fluid flow simulations. The generalized wall boundary conditions [3] are modified in order to be well adapted to multiphase fluid flows with different density and viscosity. A particle redistribution strategy is proposed R1: as an extension of the damping technique presented in [3] to adequately initialize the conditions of gravitational multiphase fluid flows. This strategy is based on the gradual application of a damping technique to mitigate gravity force in both momentum and pressure wall boundary condition equations. Several computational tests are investigated to show the accuracy and convergence of the proposed SPH interfacial multiphase model. Moreover, a simulation of a rising bubble crossing two stratified fluid layers is performed with more challenging constraints such as high density ratio, high viscosity ratio, and with presence of triple points
\end{abstract}

Email address: abdelkader.krimi@ensam.eu (Abdelkader Krimi) 
junction, and compared with the experimental results of Bonhomme et al [4]. Finally, we show the interaction between two rising bubbles test.

Keywords: Smoothed Particle Hydrodynamics, multiphase fluid flow, interfacial fluid flow, surface tension formulation, damping initial condition, Predictor-Corrector integration scheme, High density and viscosity ratio

\section{Highlights}

- A consistent SPH model was proposed to deal with interfacial multiphase fluid flow simulations;

- Robust non-conservative surface tension formulation that respects its tangential character to deal with two and more interfacial fluid phases;

- Validation of the proposed SPH model via the application on several challenging test cases of multiphase fluid flows;

- Experimental verification of applicability and accuracy of the proposed SPH interfacial multiphase model;

\section{Introduction}

In recent decades, modeling of multiphase fluid flows has been taken a large interest of researchers, scientists and engineers, due to its large applications in natural and industrial fields. Numerical models in both Mesh-based and Meshless-based approaches are available to deal with multiphase fluid flows simulations problems via the discretization of Navier-Stokes equations. In the context of grid-based approach,techniques for the capturing or tracking of interface phases are usually required. The most common techniques include the VOF (Volume Of Fluid) method [5], the LS (level set) method [6] and the front tracking method [7] [8]. The main drawback of these techniques is the difficulty to predict the evolution of a moving interface. The inaccurate prediction of the evolution of interface phases causes subsequently a wrong approximation of its curvature and normal vector which explicitly causes errors in terms of surface tension forces. The use of adaptive mesh refinement algorithms [9] can minimize these errors. However, the generation of a large amount of grid cells in these methods leads to a large computational time. The Smoothed Particle Hydrodynamics (SPH) method, is a meshless method 
that has the ability to deal with moving interfaces naturally, without using any algorithm for interface tracking. This method was first developed in 1977 to treat astrophysical simulations [10] [11]. Later, in 1988 it was applied to the simulation of fluid flows [12]. Since then, the SPH method has received lots of attention and large improvements have been developed.

In the context of SPH method, several surface tension formulations were developed to deal with multiphase fluid flows problems. Most of them are based on the continuum surface force (CSF) method developed by Brackbill et al [1] or with its variant, the Continuum Stress Surface (CSS) method [13]. For more details please refer to the Appendix A). CSF and CSS methods were initially developed for mesh-based applications. Morris [14] extended these formulations to meshless applications in the framework of the SPH method. Hu and Adams reported in [15] that when the CSS formulation is applied, a negative pressure contribution to the surface stress according to the momentum equation is observed. This negative pressure might cause instability problems near to the fluids interface.R1: Therefore, $\mathrm{Hu}$ and Adams [15] have evaluated this contribution of negative pressure and proposed a modification to the CSS formulation in order to eliminate it. Afterwards, this formulation was applied to many multiphase fluid flows applications [16][17] [18] [19] [20]. However, as it is reported in [21], this formulation does not fulfill the tangential character of the surface stress tensor (capillary pressure tensor). For more details about the tangential character please refer to Appendix B. An alternative formulation of the surface tension for SPH was presented in [22] [23] [24]. This formulation consider SPH particles as real fluid particles with attractive/repulsive forces among them. Defining these forces to reproduce the effects of surface tension obtained promising results in several test cases involving drops and flow through fractured media.

In this work, we present an extension of the surface tension formulation proposed by Adami et al [2] to be applicable in the case of more than two fluid phases simulations. It is based on the Continuum Stress Surface formulation (CSS) [1]. On the one hand, the proposed formulation enhances the stability by using a first order consistency approximation to calculate the divergence operator. On the other hand, this formulation respects the physical tangential character (as it is shown in appendix B). Similarly to [2], in this formulation the effect of the surface tension is added as a body force. The proposed formulation benefits of all the advantages of the formulation of Adami et al [2], and in addition, it can be applied to simulations with 
more than two fluid phases. These advantages make our surface tension formulation as a good alternative to the one proposed by Adams and $\mathrm{Hu}$ [15] which is widely used in the context of SPH method [16][17] [18] [19] [20]. However, and similarly to [2], the formulation does not conserve exactly the total momentum.

Moreover, we present an extension of the damping technique presented in [3] to allows for simulations of multiphase gravitational interfacial fluid flows. This technique treats the jump in initial pressure condition via the acceleration and redistribution of the initial particle distribution. This procedure has a stabilization effect during the simulation process. In addition, we also present a modification of generalized wall boundary conditions for using the SPH method in multi-phase fluid flows.

A series of benchmarks are performed to show the efficiency of the proposed formulation. These benchmarks are organized on three categories test: the first category is dedicated to test the consistency and the convergence of the proposed non-conservative surface tension force formulation. The second one investigates the ability of the presented SPH model to simulate multiphase fluid flows caused by the gravity acceleration effect (gravitational multiphase fluid flows). The third category regroups the first two ones, with some more challenging physical constraints (high ratio of density and viscosity and presence of triple point junction [25]). The obtained results show good agreement comparing with the analytical, numerical, and experimental ones available in literature.

\section{Multiphase model}

\subsection{Governing equations}

In this work we assume a weakly compressible viscous fluid flow in isothermal conditions. Under these hypothesis, the Navier-Stokes and displacement equations expressed in Lagrangian form read as

$$
\left\{\begin{array}{l}
\frac{\mathrm{d} \rho}{\mathrm{d} t}=-\rho \nabla \cdot \mathbf{v} \\
\frac{\mathrm{d} \mathbf{v}}{\mathrm{d} t}=\frac{1}{\rho}\left(-\nabla p+\mathbf{F}^{V i s}+\mathbf{F}^{S T}\right)+\mathbf{g} \\
\frac{\mathrm{d} \mathbf{r}}{\mathrm{d} t}=\mathbf{v}
\end{array}\right.
$$

where $\frac{\mathrm{d}(.)}{\mathrm{d} t}$ represents the material derivative following an infinitesimal fluid element. $\nabla$ is the nabla operator (gradient), $\rho, p, \mathbf{v}, \mathbf{r}$ and $\mathbf{g}$ represent density, 
pressure, velocity vector, position vector, and the gravitational acceleration vector, respectively. $\mathbf{F}^{\mathbf{V i s}}$ and $\mathbf{F}^{\mathbf{S T}}$ denote the viscous and surface tension forces, respectively.

The weakly compressible smoothed particle hydrodynamics approach (WC$\mathrm{SPH}$ ) was used in this work [26]. In order to close the system (1)it is required the use of an equation of state (EOS) which explicitly defines the pressure from the density instead to solve the Poisson equation. In this work the isothermal equation of state [14] is used which is expressed as

$$
p=p_{r}\left\{\left(\frac{\rho}{\rho_{0}}\right)-1\right\}+p_{b}
$$

where $\rho_{0}, p_{r}$ and $p_{b}$ denote the reference density, the reference pressure, and the background pressure, respectively. For the linear constitutive equation of state given by equation(2) the reference pressure is a function of the reference density and reference speed of sound $c_{0}$

$$
p_{r}=\rho_{0} c_{0}^{2}
$$

The use of the physical speed of sound $\mathbf{R} 2: c_{p h y}$ as a reference leads to a very small time step according to the stability conditions explained in 2.4, which causes a very large computational time. It is then a common practice to use an artificial speed of sound as a reference. Thus, following [14] and [27] the value of $c_{0}$ is determined here as

$$
c_{0}^{2} \geq \max \left\{\frac{U_{0}^{2}}{\delta \rho}, \frac{\|\mathbf{g}\| L_{0}}{\delta \rho}, \frac{\sigma}{\rho_{0} L_{0} \delta \rho}, \frac{\mu U_{0}}{\rho_{0} L_{0} \delta \rho}\right\}
$$

Where $U_{0}, L_{0}, \mu$ and $\sigma$ are the reference velocity, reference length, dynamic viscosity and surface tension coefficient, respectively. $\delta \rho$ denote the dimensionless density variation which is set to $1 \%(\delta \rho=0.01)$.

In the case of multiphase fluid flows, the reference pressure is chosen to be identical for all fluid phases, following [28]. Therefore, the speed of sound in each phase will be different in such a way that the reference pressure for all fluid phases will be conserved.

$$
p_{r}=p_{r_{1}}=\ldots=p_{r_{N_{f}}}
$$




$$
p_{r}=\rho_{0_{1}} c_{0_{1}}^{2}=\ldots=\rho_{0_{N_{f}}} c_{0_{N_{f}}}^{2} .
$$

where the subscript $N_{f}$ denotes the number of fluid phases. This condition enhances the numerical stability of the computations [28]. Hence, the choice of the artificial speed of sound $c_{0}$ is taken in such a way that both equations 4 and 6 are satisfied in all fluid phases.

For numerical problems involving single-phase free surface fluid flows, the background pressure is generally set to zero $\left(p_{b}=0\right)$. Furthermore, for simulations of single or multiphase confined fluid flows, the pressure is chosen as a positive value sufficient to guarantee the positivity of the calculated pressure field via the equation of state in order to avoid the tensile instability [29]. In this work, the numerical experiments show that the ideal background pressure $p_{b}$ is chosen as a function of the reference pressure and it is proportional to $0.05 p_{r}$ (i.e $: p_{b} \propto 0.05 p_{r}$ ).

\subsection{Discrete form of governing equations}

The smoothed particle hydrodynamics is a meshless method. It discretizes the physical space into many R1: discrete elements, usually called particles, without any connectivity among them. This method is based on the approximation of any physical scalar (or vector) field using the convolution formulation. Numerically, it is performed by replacing the Dirac delta function with a regular smooth function, which is called kernel. This function must satisfy some conditions such as symmetry (even function), normalization, compactness of it support, among others. We refer the interested reader to [30] for more details. The kernel function used in this work is the quintic spline [31] (equation 7) . This kernel was selected since it prevents a high disorder in the particle distribution. The kernel function depends on a parameter $h$, called the smoothing length, which defines the domain of influence of the kernel function. In this work, the smoothing length $h$ is a constant which is chosen relative to the initial inter-particle distance $\delta x_{0}\left(h=1.33 \delta x_{0}\right)$. The initial particle volume is taken as $V_{0}=\delta x_{0}{ }^{d}$, with $d$ is the space dimension number. The mass of each particle $i$ of different fluid phases is chosen to be constant and equal to $m=\rho_{0_{\text {Phase }}} V_{0}$ during all the simulation time. 


$$
W(r, h)=\alpha_{d}\left\{\begin{array}{cl}
\left(3-\frac{r}{h}\right)^{5}-6\left(2-\frac{r}{h}\right)^{5}+15\left(1-\frac{r}{h}\right)^{5} & 0 \leq \frac{r}{h}<1 \\
\left(3-\frac{r}{h}\right)^{5}-6\left(2-\frac{r}{h}\right)^{5} & 1 \leq \frac{r}{h}<2 \\
\left(3-\frac{r}{h}\right)^{5} & 2 \leq \frac{r}{h}<3 \\
0 & \frac{r}{h} \geq 3
\end{array}\right.
$$

where $\alpha_{d}=\frac{7}{478 h^{2} \pi}$ for $2 \mathrm{D}$ cases, and $r$ is the distance between two neighboring particles $i$ and $j\left(r=r_{i j}=\left\|\mathbf{r}_{\mathbf{i}}-\mathbf{r}_{\mathbf{j}}\right\|\right)$.

$\mathrm{Hu}$ and Adams [15] developed a formulation that exactly guarantees mass conservation. In this formulation, the continuity equation of the NavierStokes system (1), can be replaced by the expression

$$
\rho_{i}=m_{i} \sum_{j}^{n_{b}} W_{i j}
$$

where $\rho_{i}$ and $m_{i}$ are the density and the mass of the particle $i$, respectively. $W_{i j}=W\left(\mathbf{r}_{\mathbf{i j}}, h\right)$ is the Kernel function, $\mathbf{r}_{\mathbf{i j}}=\mathbf{r}_{\mathbf{i}}-\mathbf{r}_{\mathbf{j}}$ is the distance between the particle $i$ and its neighbours $j$. The number of particles in the neighborhood of particle $i$ is denoted as $n_{b}$.

This formulation is widely used in SPH codes, and it works very well in the case of confined fluid flow simulations and allows the use of higher values of the CFL number. However, this formulation is very sensitive to the particle disorder. Subtle variations in the particle positions cause high fluctuations in the particle density and thus in pressure specially in the case of gravitational fluid flows. This problem can be alleviated by an adequate redistribution and acceleration of the initial particle positions and velocities by using a damping technique that will be detailed in Section 2.5.

$$
\frac{\mathrm{d} \mathbf{v}_{\mathbf{i}}}{\mathrm{d} t}=\frac{1}{\rho_{i}}\left(-\nabla p_{i}+\mathbf{F}_{\mathbf{i}}^{V i s}+\mathbf{F}_{\mathbf{i}}^{S T}\right)+\mathbf{g}_{\mathbf{i}}
$$

The acceleration of the particle $i$ due to the gradient of pressure is approximated following the work in [2] as

$$
\frac{1}{\rho_{i}} \nabla p_{i}=\frac{1}{m_{i}} \sum_{j}^{n_{b}}\left(V_{i}^{2}+V_{j}^{2}\right) \widetilde{p_{i j}} \nabla W_{i j}
$$


Where $V_{i}=\frac{m_{i}}{\rho_{i}}$ is the volume of particle $i$. The term $\left(\nabla W_{i j}=\frac{\partial W}{\partial r_{i j}} \mathbf{e}_{\mathbf{i j}}\right)$ is the gradient of the kernel function, and $\mathbf{e}_{\mathbf{i j}}=\frac{\mathbf{r}_{\mathbf{i j}}}{r_{i j}}=\frac{\mathbf{r}_{\mathbf{i}}-\mathbf{r}_{\mathbf{j}}}{r_{i j}}$ is the unit inter-particle vector.

The term $\widetilde{p_{i j}}$ is defined to ensure the continuity of pressure even for the case of discontinuous density between fluid particles (for example, when they belongs to different phases). Following [32] this term reads as

$$
\widetilde{p_{i j}}=\frac{\rho_{j} p_{i}+\rho_{i} p_{j}}{\rho_{i}+\rho_{j}}
$$

The acceleration due to the viscous forces can be expressed as in [2]

$$
\frac{1}{\rho_{i}} \mathbf{F}_{\mathbf{i}}{ }^{V i s}=\frac{1}{m_{i}} \sum_{j}^{n_{b}}\left(V_{i}^{2}+V_{j}^{2}\right) \widetilde{\mu_{i j}} \frac{\mathbf{v}_{\mathbf{i j}}}{r_{i j}} \frac{\partial W}{\partial r_{i j}}
$$

Where $\mathbf{v}_{\mathbf{i j}}=\mathbf{v}_{\mathbf{i}}-\mathbf{v}_{\mathbf{j}}$ is the relative velocity between the particle $i$ and $j$. The term $\widetilde{\mu_{i j}}$ is the inter-particle-averaged dynamic viscosity which is defined as

$$
\widetilde{\mu_{i j}}=\frac{2 \mu_{i} \mu_{j}}{\mu_{i}+\mu_{j}}
$$

In equation (13), $\mu_{i}$ is the dynamic viscosity of the particle $i$.

This form of viscous acceleration conserves the linear momentum [2], and performs well in the case of short-time simulations. In this work we have chosen an alternative formulation [33] which conserves both angular and linear momentum in order to perform long-time simulations. This alternative formulation reads as

$$
\frac{1}{\rho_{i}} \mathbf{F}_{\mathbf{i}}{ }^{V i s}=\frac{\zeta}{m_{i}} \sum_{j}^{n_{b}}\left(V_{i}^{2}+V_{j}^{2}\right) \widetilde{\mu_{i j}} \frac{\mathbf{v}_{\mathbf{i j}} \mathbf{r}_{\mathbf{i j}}}{r_{i j}^{2}} \nabla W_{i j}
$$

Where $\zeta=d+2$, and $d$ is the space dimension number.

\subsubsection{The surface tension force:}

In the case of a two-phase fluid, a Continuum Surface Force (CSF) formulation [1] may be used to represent the surface tension force. This formulation describes the pressure-jump condition normal to the separation interface of 
the fluids. Assuming that the surface tension coefficient $\sigma^{(1-2)}$ is constant between two fluid phases ( 1 and 2), the expression of the force can be expressed as

$$
\mathbf{F}^{S T_{(1-2)}}=-\sigma^{(1-2)} \kappa \mathbf{n} \delta_{\Sigma}
$$

In equation (15), $\kappa, \mathbf{n}$ and $\delta_{\Sigma}$ denote the curvature, the normal vector to the interface (see figure 17) and the delta function, respectively. In the context of the SPH method, equation (15) reads as

$$
\mathbf{F}_{i}^{S T_{(1-2)}}=-\sigma^{(1-2)} \nabla \cdot \mathbf{n}_{i} \nabla C
$$

where $C$ is the color function that has a unit jump across the interface. It's equal to 1 in one particle fluid phase and 0 in its neighboring particle of other fluid phase.

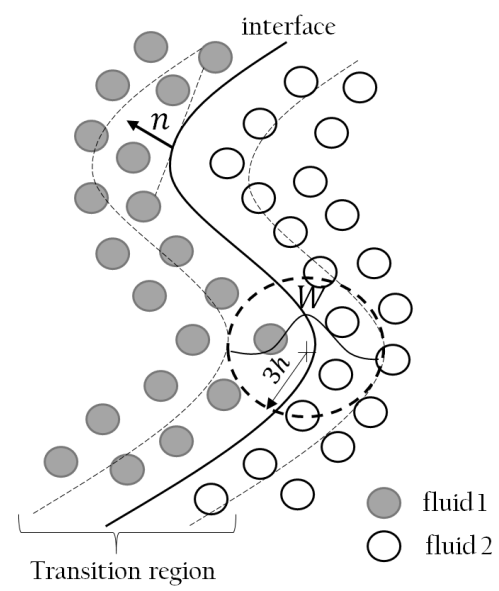

Figure 1: Geometrical description of the principal parameter of surface tension formulation in the case of two immiscible fluids 1 and 2: interface, transition region, normal vector $\mathbf{n}$. The thickness of the transition region is equivalent to the diameter of the kernel function ( $6 h$ for the quintic kernel function)

The previous formulation for the surface tension force is applicable in the case of two-phase fluid flows. If more than two phases are present in the flow, the explicit use of this formulation becomes impractical in the context of SPH method. 
Thus, an alternative formulation should be used in these cases. The Continuum Surface Stress (CSS) [13] is a tensorial formulation of the surface tension force equivalent to the CSF formulation given in equation (16). The CSS formulation can be expressed as a body force applied through a transition region of finite thickness. The size of this finite thickness is equal to the diameter of Kernel function (see figure 1)

$$
\mathbf{F}_{i}^{S T}=\nabla \cdot \boldsymbol{\Pi}_{\mathbf{i}}
$$

where $\boldsymbol{\Pi}_{\mathbf{i}}$ defines the immiscible mixture surface stress tensor of the particle $i$ (capillary pressure tensor). Assuming that the particle $i$ belongs to the $l$ fluid phase, then the mixture surface stress can be expressed as

$$
\Pi_{\mathbf{i}}=\sum_{k \neq l} \Pi_{\mathbf{i}}^{\mathrm{kl}}
$$

In equation $18, \boldsymbol{\Pi}_{\mathbf{i}}^{\mathbf{k l}}$ is the fluid surface stress tensor between phases $k$ and $l$, defined as

$$
\mathbf{\Pi}_{\mathbf{i}}^{\mathbf{k l}}=\frac{\sigma^{k l}}{\left\|\nabla C_{i}^{k l}\right\|}\left(\left\|\nabla C_{i}^{k l}\right\|^{2} \mathbf{I}-\nabla C_{i}^{k l} \otimes \nabla C_{i}^{k l}\right)
$$

By assuming that the particle $i$ belongs to the phase $l$, the gradient of the color function at the interface between two different phases $k$ and $l\left(\nabla C_{i}^{k l}\right)$ reads as [2]

$$
\nabla C_{i}^{k l}=\frac{1}{V_{i}} \sum_{j}^{n_{b}}\left(V_{i}^{2}+V_{j}^{2}\right) \widetilde{C_{i j}^{k l}} \nabla W_{i j}
$$

The inter-particle-averaged color function $\widetilde{C_{i j}^{k l}}$ is defined as

$$
\widetilde{C_{i j}^{k l}}=\left\{\begin{array}{cl}
\frac{\rho_{i}}{\rho_{i}+\rho_{j}} & \text { if the particle } j \text { belongs to the } k \text { fluid phase } \\
0 & \text { Otherwise }
\end{array}\right.
$$

The CSS formulation given by equations (18) and (19) is a variation of the CSF formulation (equation (15)). The relation between the CSF and the CSS formulations is presented in the Appendix A. In the CSS formulation, the interior efforts associated to the surface stress tensor $\Pi$ are tangent to 
the interface, which is coherent with the propriety of surface tension force (the details are presented in the Appendix B ) The direct application of the CSS formulation given in equation (17) in the context of the SPH method, could lead to numerical instabilities [14, 15, 2].

R1: In this work, the divergence formulation used in [2] for the calculation of the curvature of the interface is adopted to calculate the divergence of the capillary pressure tensor (equation (17)). This formulation does not require a matrix inversion and gives a first-order consistency approximation. Or in other words, This formulation can reproduce exactly the divergence of any linear field. Thus, the modified discrete form of equation (17) is

$$
\mathbf{F}_{i}^{S T}=d \frac{\sum_{j}^{n_{b}} V_{j} \boldsymbol{\Pi}_{\mathbf{i j}} \nabla W_{i j}}{\sum_{j}^{n_{b}} V_{j} r_{i j} \frac{\partial W}{\partial r_{i j}}}
$$

where we define the inter-particle surface stress tensor as $\boldsymbol{\Pi}_{\mathbf{i j}}=\boldsymbol{\Pi}_{\mathbf{i}}-\boldsymbol{\Pi}_{\mathbf{j}}$, d is the number of space dimensions and $r_{i j}=\left\|\mathbf{r}_{\mathbf{i}}-\mathbf{r}_{\mathbf{j}}\right\|$ is the inter-particle distance.

R1: Note that with this formulation, the surface tension force does not exactly conserve the total momentum. However, It grantees a good approximation even when a disordered particles distribution is presented or the support of the kernel function is not full with particles contained within the transition region. This force takes the effect as a body one. This formulation takes all advantageous of the formulation proposed in [2], and in addition it can be applicable in the case of more than two-phases fluid flows simulation problems.

\subsection{Wall boundary conditions}

In this work, we also present a modification of the generalized wall boundary condition method proposed by [3] to deal with multiphase fluid flows. In this method, three layers of dummy particles must be added in the normal direction to the wall interface (see figure 2). The dummy particles are placed to represent the wall in such a way that it is ensured the completeness of the support of kernel function, in order to obtain an accurate integration of the 
field variables near the wall interface. Free-slip or no-slip wall boundary conditions can be applied using this method. The free-slip boundary condition is applied by omitting the viscous interaction between the fluid particle with the adjacent dummy particles in the calculation of fluid viscous forces (equation 14). In the case of no-slip wall boundary condition, a virtual velocity $\mathbf{v}_{\mathbf{w}}$ is imposed to the wall-dummy particle interacting with the fluid particle $i$ in equation 14 . This velocity is defined as

$$
\mathbf{v}_{\mathbf{w}}=2 \mathbf{v}_{\mathbf{i}}-\tilde{\mathbf{v}}_{\mathbf{i}}
$$

where $\mathbf{v}_{\mathbf{i}}$ is the prescribed velocity of wall particle $i$ and $\tilde{\mathbf{v}}_{\mathbf{i}}$ denotes the interpolation of the smoothed velocity field of the fluid phase to the dummy particle position. The term $n_{f}$ refers to the number of neighboring fluid particles $j$ of the wall particle $i$.

$$
\tilde{\mathbf{v}}_{\mathbf{i}}=\frac{\sum_{j}^{n_{f}} \mathbf{v}_{\mathbf{j}} W_{i j}}{\sum_{j}^{n_{f}} W_{i j}}
$$

The pressure in the dummy-wall particle is calculated from the neighboring fluid particles $j$ according to [3]

$$
p_{w}=\frac{\sum_{j}^{n_{f}} p_{j} W_{w j}+\left(\mathbf{g}-\mathbf{a}_{\mathbf{w}}\right) \sum_{j}^{n_{f}} \rho_{j} \mathbf{r}_{\mathbf{w j}} W_{w j}}{\sum_{j}^{n_{f}} W_{w j}}
$$

where the term $\mathbf{a}_{\mathbf{w}}$ represents a prescribed wall acceleration, if moving walls are present.

The method proposed in [3] computes the density of the dummy particle via the equation of state. This formulation is applicable for the case of single fluid with constant viscosity or in case of multiphase inviscid flows [34] [35] [36]. In the case of multiphase viscous flows where different viscosities are associated to each phase, this formulation becomes unusable because of the ambiguity in the choice of the viscosity for the wall-dummy particles. This is specially 


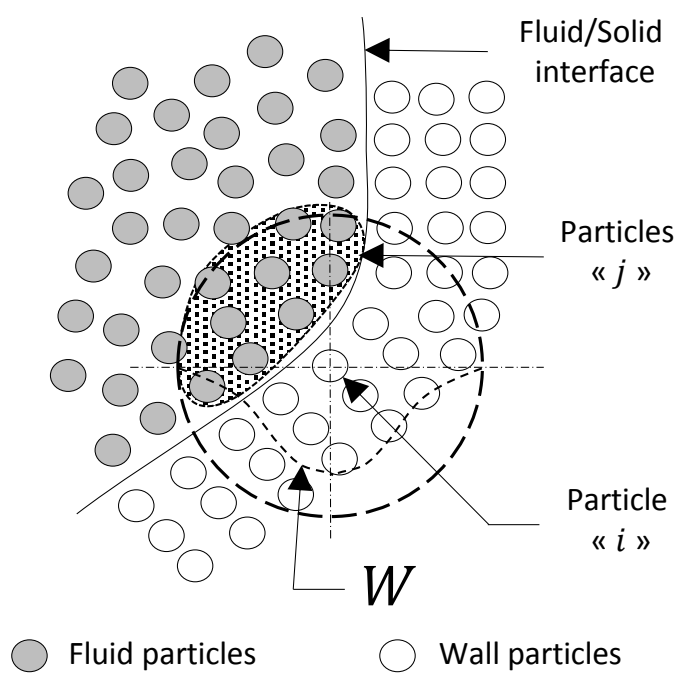

Figure 2: Geometrical description of different parameters used in the generalized wall Boundary condition. Figure based on that presented in [3]

noted when the neighboring particles belong to different fluid phases. Here we solve this ambiguity by using a method based on the fluid particle mirror similarity. We assume that each fluid particle considers all their wall-dummy neighbor particles as similar to it in terms of density, viscosity and volume. Using this approach we need to modify equations (10), (12) or (14) as follows

$$
\begin{gathered}
\frac{1}{\rho_{i}} \nabla p_{i}=\frac{1}{m_{i}} \sum_{j}^{n_{b}} \widetilde{\mathcal{P}_{i j}^{V}} \nabla W_{i j} \\
\frac{1}{\rho_{i}} \mathbf{F}_{\mathbf{i}}^{V i s}=\frac{1}{m_{i}} \sum_{j}^{n_{b}} \widetilde{\mu_{i j}} V \frac{\mathbf{v}_{\mathbf{i j}}}{r_{i j}} \frac{\partial W}{\partial r_{i j}} \\
\frac{1}{\rho_{i}} \mathbf{F}_{\mathbf{i}}^{V i s}=\frac{\zeta}{m_{i}} \sum_{j}^{n_{b}} \widetilde{\mu_{i j}} V \frac{\mathbf{v}_{\mathbf{i j}} \mathbf{j}_{\mathbf{i j}}}{r_{i j}^{2}} \nabla W_{i j}
\end{gathered}
$$

Where $\mathcal{P}_{i j}^{V}$ and ${\widetilde{\mu_{i j}}}^{V}$ are defined as

$$
\widetilde{\mathcal{P}_{i j}^{V}}=\left\{\begin{array}{cl}
\left(V_{i}^{2}+V_{j}^{2}\right) \frac{\rho_{j} p_{i}+\rho_{i} p_{j}}{\rho_{i}+\rho_{j}} & \text { if the particle } j \text { is a fluid particle } \\
V_{i}^{2}\left(p_{i}+p_{j}\right) & \text { if the particle } j \text { is a wall particle }
\end{array}\right.
$$




$$
\widetilde{\mu_{i j}} V=\left\{\begin{array}{cl}
2\left(V_{i}^{2}+V_{j}^{2}\right) \frac{\mu_{i} \mu_{j}}{\mu_{i}+\mu_{j}} & \text { if the particle } j \text { is a fluid particle } \\
2 V_{i}^{2} \mu_{i} & \text { if the particle } j \text { is a wall particle }
\end{array}\right.
$$

Note that the direct application of the proposed wall boundary conditions method can present spurious currents when the interfaces between the fluid phases includes a surface tension next to the wall boundaries. To deal with this issue, a special treatment as proposed in [37] can be added to this method. However, this problem is not addressed here since it is out of the scope of the present work.

\subsection{Time stepping}

R1: In this work a Predictor-Corrector scheme is proposed for time integration. An explicit Euler method is used to predict the velocity $\left(\widetilde{\mathbf{v}}_{\mathbf{i}}^{n+1}\right)$ and the position $\left(\widetilde{\mathbf{r}}_{\mathbf{i}}^{n+1}\right)$ of the particle $i$.

$\mathbf{R} 1:$ The corrected velocity at $n+1\left(\mathbf{v}_{\mathbf{i}}{ }^{n+1}\right)$ is approximated by using trapezoidal-rule, using predicted parameters (.) ${ }^{n+1}$ instead of the final ones $(.)^{n+1}$. Note that, the density and pressure at time $n\left(\rho^{n}, p^{n}\right)$ and the predicted values of velocity and position $\left(\widetilde{v}^{n+1}, \widetilde{r}^{n+1}\right)$ are used to predict the right hand-side of momentum equation $\left(\frac{\widetilde{\mathrm{d} \mathbf{v}_{\mathbf{i}}}}{\mathrm{d} t}\right)^{n+1}$. The final position $\mathbf{r}_{\mathbf{i}}{ }^{n+1}$ is advected by the corrected velocity.

The following algorithm summarizes the prediction step.

$$
\left\{\begin{array}{l}
\widetilde{\mathbf{v}}_{\mathbf{i}}^{n+1}=\mathbf{v}_{\mathbf{i}}^{n}+\delta t\left(\frac{\mathrm{d} \mathbf{v}_{\mathbf{i}}}{\mathrm{d} t}\right)^{n} \\
\widetilde{\mathbf{r}}_{\mathbf{i}}^{n+1}=\mathbf{r}_{\mathbf{i}}^{n}+\delta t\left(\frac{\mathrm{d} \mathbf{i}}{\mathrm{d} t}\right)^{n}
\end{array}\right.
$$

and the correction step is summarized as follows

$$
\left\{\begin{array}{l}
\mathbf{v}_{\mathbf{i}}^{n+1}=\mathbf{v}_{\mathbf{i}}^{n}+\frac{\delta t}{2}\left\{\left(\frac{\mathrm{d} \mathbf{v}_{\mathbf{i}}}{\mathrm{d} t}\right)^{n}+\left(\frac{\widetilde{d \mathbf{v}_{\mathbf{i}}}}{\mathrm{d} t}\right)^{n+1}\right\}=\frac{1}{2}\left\{\mathbf{v}_{\mathbf{i}}^{n}+\widetilde{\mathbf{v}}_{\mathbf{i}}^{n+1}\right\}+\frac{\delta t}{2}\left(\frac{\widetilde{\mathrm{d}} \mathbf{v}_{\mathbf{i}}}{\mathrm{d} t}\right)^{n+1} \\
\mathbf{r}_{\mathbf{i}}^{n+1}=\mathbf{r}_{\mathbf{i}}^{n}+\delta t \mathbf{v}_{\mathbf{i}}^{n+1}
\end{array}\right.
$$

The final density (at time $n+1$ ) is calculated as $\rho_{i}^{n+1}=m_{i} \sum_{j}^{n_{b}} W\left(\widetilde{\mathbf{r}_{\mathrm{ij}}}\right)^{n+1}$. Afterwards, the final pressure $p^{n+1}=p\left(\rho^{n+1}\right)$ is calculated according to the equation of state $(2), p^{n+1}=p\left(\rho^{n+1}\right)$. 
The superscripts $n$ and $n+1$ refers to the time step, whereas $\widetilde{\{.\}}$ refers to the predicted physical parameter $\{$.$\} . For more details about the use of$ this scheme in the context of interfacial multiphase SPH model please see Appendix C.

To ensure the stability of the method, the time step $(\delta t)$ must be chosen to fulfill the kinetic, viscous, body force and surface tension conditions [35] [1]

$$
\begin{gathered}
\delta t=C F L \frac{h}{\max \left(c_{0_{i}}\right)+\max \left(\left\|\mathbf{v}_{\mathbf{i}}\right\|\right)} \\
\delta t \leq 0.125 \frac{h^{2}}{\max \left(\frac{\mu_{i}}{\rho_{0_{i}}}\right)} \\
\delta t \leq 0.25\left(\frac{h}{\|\mathbf{g}\|}\right)^{1 / 2} \\
\delta t \leq 0.25\left(\frac{\min \left(\rho_{k}, \rho_{l}\right) h^{3}}{2 \pi \sigma^{k-l}}\right)^{1 / 2}
\end{gathered}
$$

By using the density summation formulation (equation 8) with this time integration scheme, the simulations were stable with CFL numbers equal to one. In the numerical simulations presented here, a value of $C F L=1$ is employed.

\subsection{Damping strategy for multiphase fluid flow}

In the framework of weakly compressible fluid flows, the accuracy on the determination of the pressure field using an equation of state depends on the density estimation. Here, the density of the particles is updated using the equation (8). It becomes obvious that a good estimation of the position of the particles is crucial to obtain a good approximation of the pressure.

In this work, an initial regular lattice distribution of particles is chosen to perform the simulation. However, in the context of gravitational fluid flow problems, the use of the density summation formulation (equation (8)) to update density (and thus the pressure with equation (2)) in a regular distribution particles may spoil the imposed initial hydrostatic pressure and cause spurious high-frequency oscillations. In single fluid flow formulations, such artifacts can be reduced using a damping technique during the initial transient of simulations $[3,38]$. This damping smooths both the distribution 
and the velocity of the particles to mitigate the oscillations. In fact, we introduce a mitigation factor $\left(\xi_{D}(t) \leq 1\right)$ which acts as a multiplication factor on the body force in the momentum equation (9) as well as in the wall pressure equation 25, to obtain a gradual introduction of the gravity force. The mitigation factor is only activated during the time $T_{D}$ (damping time), and is defined as

$$
\xi_{D}(t)=\left\{\begin{array}{cc}
\left(\sin \left(\frac{t}{T_{D}}-0.5\right) \pi+1\right) & t \leqslant T_{D} \\
1 & t>T_{D}
\end{array}\right.
$$

Note that other expressions are possible instead of 37, as for example, the Hill equation [39].

Unfortunately, these damping techniques [3, 38] are not applicable in the case of the simulation involving gravitational multiphase flows. This is due to the difference in density between the fluid phases (buoyancy force), which generates a considerable motion of the particles during the damping period. To extend the application of this technique to gravitational multiphase fluid flow simulations, a new strategy must be defined. In this work, all the physical properties of all fluid phases (reference density, viscosity, mass ...) are set to be equal to those of the heavier phase during the damping procedure, in order to avoid any motion due to the different properties between the phases. This technique allows the particles to be slightly redistributed and accelerated in order to reach a good estimation of the initial hydrostatic pressure and velocity. After the damping time, the real physical properties must be assigned again to each phase, and the calculation procedure continues as usual. The reader is referred to Appendix $\mathrm{C}$ for more details about the use of this technique in the case of interfacial multiphase fluid flow simulations.

\section{Numerical applications and validation}

In this section we present several numerical examples to test the accuracy, stability and applicability of the proposed SPH model for multiphase flow problems. Three categories of tests are investigated:

The first category of tests is dedicated to investigate the consistency and the convergence of the non-conservative formulation for the surface tension force 
proposed in this work, for the case of two and three phase flows with presence of triple junction points. This is done through the comparison with available analytical solutions. The examples addressed in this category are the square droplet deformation [2] ,the spreading lens between two stratified fluid layers [40] [25], and the capillary-viscous wave test [14, 15, 16, 17, 2] .

The second category of tests is devoted to demonstrate the ability of the presented SPH model for the simulation of multiphase flows under gravity effects neglecting the surface tension. The examples addressed here are the evolution of the two-phase stratified fluid layers and the Rayleigh-Taylor instability (RTI). The first example is not only performed to examine the stabilization effect of the damping technique but also to validate the proposed modifications on generalized wall boundary conditions when the two fluid phases meet the solid boundaries. The second example, is dawn to compare the results of the presented SPH multiphase model with the ones using LevelSet [18] and other SPH [41] models, and also with the analytical approach presented in [42].

In the third category of tests we introduce high density and viscosity ratio effects. The rising of an air bubble trough a water column and through two stratified fluid layers are investigated. The results obtained with the new method are compared to those obtained with other numerical methods (Volume-Of-Fluid [43], Level-Set [44]) and also with experimental results [4]. Finally, we present the example of two rising bubbles through a water column using a higher particle resolution than in the previous examples. In all the numerical examples of this paper, the measurement of the physical properties obtained at any desired point $\mathbf{r}_{\mathbf{m}}$ has been performed using a zeroth order consistency SPH approximation (Shepard filter [45])

$$
p\left(\mathbf{r}_{\mathbf{m}}\right)=\frac{\sum_{i}^{n} V_{i} p_{i} W\left(\mathbf{r}_{\mathbf{m}}-\mathbf{r}_{\mathbf{i}}\right)}{\sum_{i}^{n} V_{i} W\left(\mathbf{r}_{\mathbf{m}}-\mathbf{r}_{\mathbf{i}}\right)}
$$

Where $n$ refers to the number of neighboring particles of the measuring point $\mathbf{r}_{\mathrm{m}}$. 
3.1. First category of tests: Validation of the formulation for the surface tension

We present here the first category of numerical tests devoted to the validation of the formulation for the surface tension.

\subsubsection{Square droplet deformation}

In this first test case, the SPH method with CSS model of the surface tension force is applied to the simulation of the deformation of a square droplet under the action of the surface tension force. This example has already been investigated by Adami et al [2] using a SPH method with CSF model. The square droplet is defined by an edge length $l_{d}=0.6[\mathrm{~m}]$ units. It is placed on the center of an square box with sides $L_{s}=1[\mathrm{~m}]$. The fluid within the square droplet is referred as phase 1 whereas the fluid outside the droplet is referred as phase 2 (see fgure 3). The densities of the each fluid phases are chosen as $\rho_{1}=\rho_{2}=1\left[\mathrm{Kg} / \mathrm{m}^{3}\right]$ and the dynamic viscosity is taken as $\mu_{1}=\mu_{2}=0.2\left[\right.$ Pa.s]. The surface tension coefficient is $\sigma^{1-2}=1[\mathrm{~N} / \mathrm{m}]$.

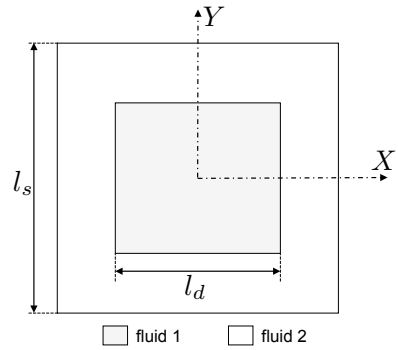

Dimensions

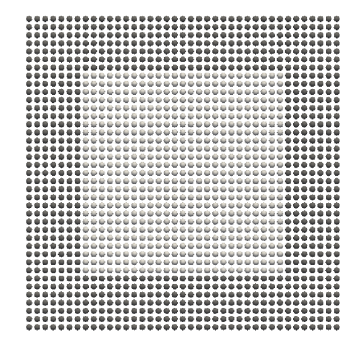

Initial state

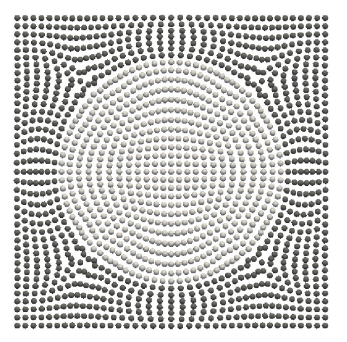

Equilibrium state

Figure 3: The evolution of square droplet under the surface tension effort : The left figure describes the geometrical details. The middle figure shows the initial fluid particles at $(t=0[s])$ for the particle resolution $\frac{l_{s}}{40}$. The right figure describes droplet in equilibrium state after its evolution for the particle resolution $\frac{l_{s}}{40}$.

We investigate the evolution of the square droplet deformation using three different number of particles: $\frac{l_{s}}{40}, \frac{l_{s}}{80}$ and $\frac{l_{s}}{160}$. The smoothing length is chosen equal to $h=1.33 \delta x_{0}$. The reference speed of sound is set to $\left(c_{0}=10[\mathrm{~m} / \mathrm{s}]\right)$ for all resolutions. A positive background pressure is taken as $\left(p_{b}=5[\mathrm{~Pa}]\right)$. A non-slip boundary condition is applied on all sides of the square box.

The evolution of the kinetic energy of the particles inside the droplet $\left(E_{k}=\right.$ $\frac{1}{2} \sum_{i_{d}} m_{i_{d}}\left\|v_{i d}\right\|^{2}$ where $i_{d}$ refers to the droplet particles) is plotted in figure 
4. The deformation of the square droplet starts at the corners because of a local high surface tension due to the high curvature at corners. This effect is reflected by the peak in the kinetic energy which evolves until the stabilization in a value close to zero for all resolutions.

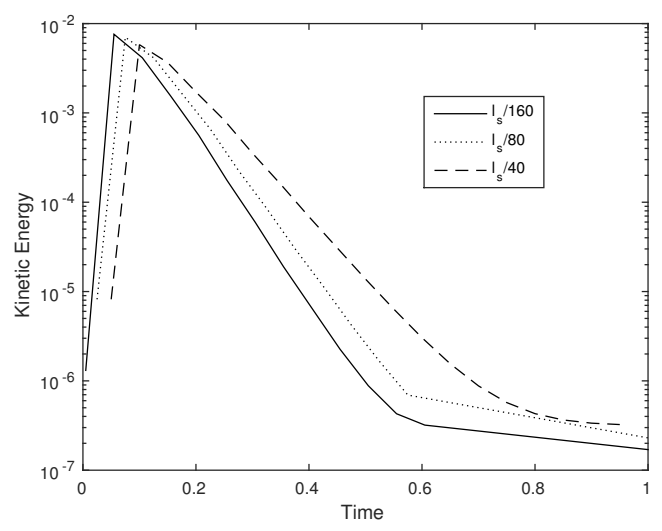

Figure 4: Time evolution of droplet kinetic energy for particle resolutions $\frac{l_{s}}{40}, \frac{l_{s}}{80}$ and $\frac{l_{s}}{160}$.

At the stabilized state (equilibrium state) the particles are in rest, and the square droplet is totally transformed in a circular droplet. Under the fluid incompressibility hypothesis, both phases must conserve their volumes (areas in 2D) during all the evolution process. Thus, the equality $\pi R^{2}=l_{d}^{2}$ holds for for the square droplet, and therefore the equilibrium radius is $R=$ $\frac{l_{d}}{\sqrt{\pi}} \approx 0.338[\mathrm{~m}]$. From Laplace's law, the pressure of the fluid particles inside the droplet (phase 1) must be higher than that of the surrounding particles (phase 2), and the jump of pressure between the two phases must satisfy the condition

$$
\Delta p=\frac{\sigma^{1-2}}{R} \approx 2.954[P a]
$$

The surface tension forces orientation and the magnitude of the velocity are shown in figure 5 . We observe that the surface tension force has a radial direction, oriented towards the center of droplet. This orientation corresponds to the direction of normal vectors to the interface between the fluid phases (see the left side of the figure 5 ). On the right side of the figure 5 the magnitude velocity of every particle is represented. The velocity magnitude 

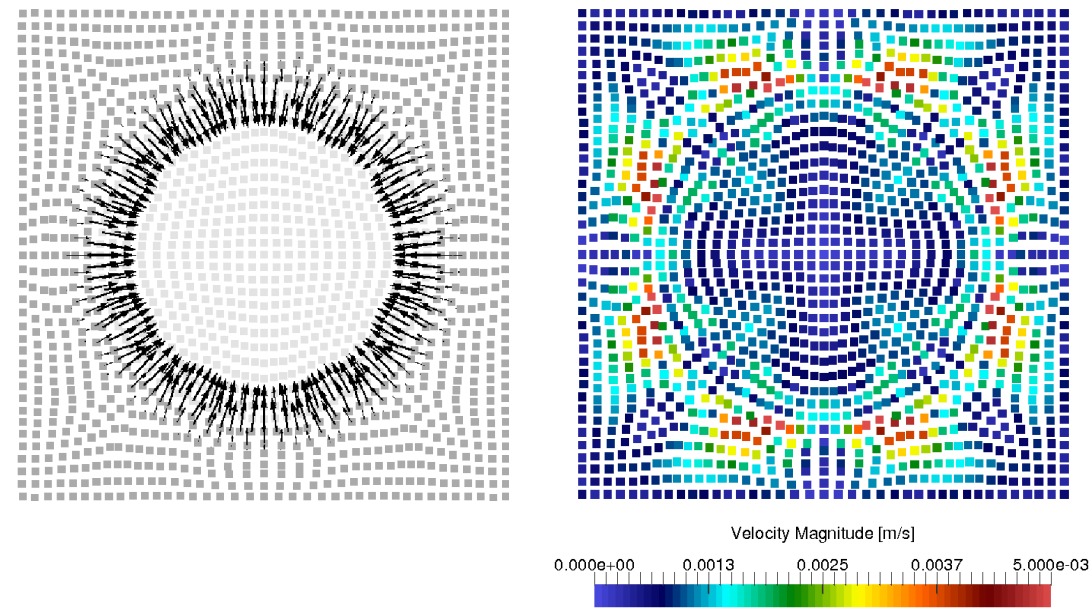

Figure 5: Surface tension forces orientation (on the left) and magnitude of the velocity (on the right) of droplet at equilibrium state for the particle resolution $\frac{l_{s}}{40}$.

is in the order of $O\left(10^{-3}\right)$. When forces due to this spurious effect are comparable to other physical forces such as viscous, gravitational, and surface tension forces, errors will be greater.

In figure 6 we show the cut of pressure field at $Y=0$ (X-axis) obtained in the simulations and also the analytical pressure predicted by Laplace's law. It is observed a good agreement between the numerical results and the theoretical pressure. These results show that the SPH method with the CSS non-conservative surface tension model is able to represent correctly the equilibrium state of this two phase flow problem.

When a low viscosity of $\mu_{1}=\mu_{2}=0.001[$ Pa.s] is used (small viscous forces) to simulate the square droplet deformation test case, the pressure profile at the equilibrium state is less accurate. This is because in this case viscosity forces are comparable with the forces due to the parasitic currents (see figures 7 and 8). Note however that the circular shape of droplet is well approximated. Parasitic currents are a numerical artifact suffered for numerical approaches of the surface tension based on the use of CSF or CSS formulations. The elimination of this effect is not addressed here. For more details about parasitic currents artifact and their elimination, please refer to $[46,47,48]$.

Three density ratios $\frac{\rho_{2}}{\rho_{1}}=\{1,10,1000\}$ are investigated in order to show the influence of the variation of density ratios on the obtained pressure re- 

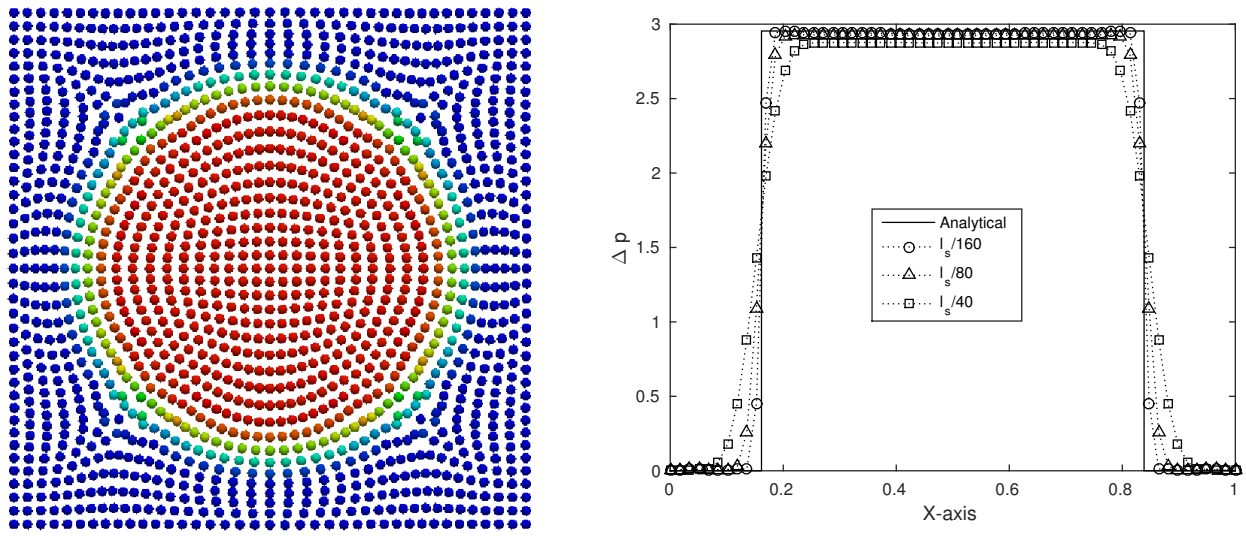

Figure 6: Square droplet test case. The left figure plots the normalized pressure $\left(\frac{p-p_{2}}{p_{1}-p_{2}}\right)$ at the final stabilized state. The figure on the right plots a cut of the pressure field at $Y=0$ obtained by the numerical method and the theoretical solution for different particle resolutions $\frac{l_{s}}{160}, \frac{l_{s}}{80}$ and $\frac{l_{s}}{40}$.
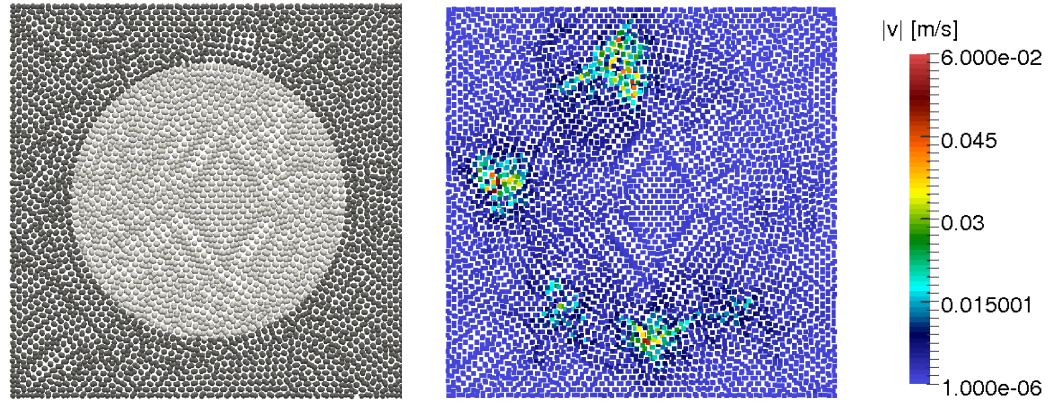

Figure 7: Square droplet test case using low viscosity $\left(\mu_{1}=\mu_{2}=0.001[\right.$ Pa.s $\left.]\right)$ for the particle resolution of $\frac{l_{s}}{80}$. Particles colored with fluid phases (left). Magnitude velocity field (right).

sults. In figure 9 , the pressure profiles at $Y=0$ are plotted for three selected density ratios. Despite the obtained results of pressure are not as accurate as for the case of density ratio $\frac{\rho_{2}}{\rho_{1}}=1$, we observe relatively a good agreement with the analytical solution for the density ratios of $\frac{\rho_{2}}{\rho_{1}}=10$ and $\frac{\rho_{2}}{\rho_{1}}=1000$.

\subsubsection{Spreading lens between two stratified layers}

This example is aimed to test if the presented SPH model can deal with triple junction points problems [25]. Thus, the classical test of the spreading of a lens between two stratified fluid layers is investigated. A circular lens of 


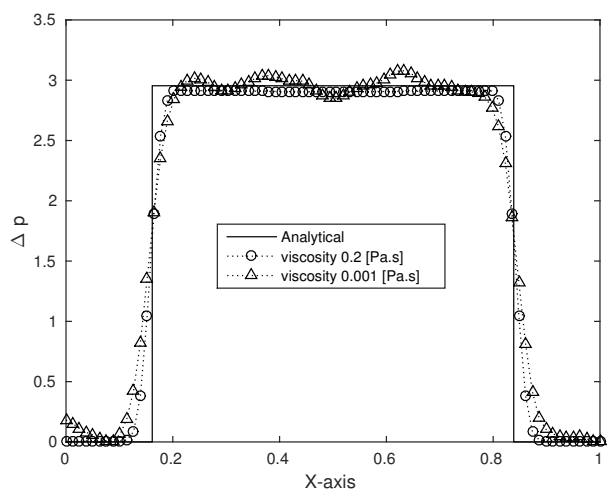

Figure 8: A cut of the pressure field at $Y=0$ obtained by the theoretical solution and the numerical method and numerical method with viscosity $\mu_{1}=\mu_{2}=0.2[$ Pa.s $]$ (dotted line with small circles) and $\mu_{1}=\mu_{2}=0.001[P a . s]$ (dotted line with small triangles) for the particle resolution of $\frac{l_{s}}{80}$.

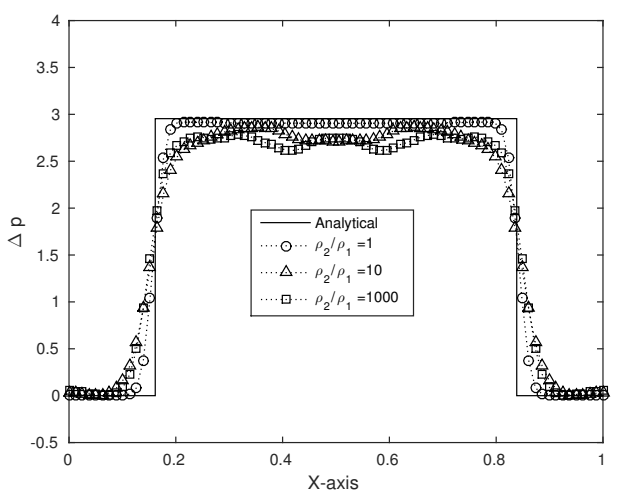

Figure 9: A cut of the pressure field at $Y=0$ obtained by the theoretical solution and the numerical method and numerical method with viscosity $\mu_{1}=\mu_{2}=0.2[$ Pa.s $]$ and three density ratios $\frac{\rho_{2}}{\rho_{1}}=\{1,10,1000\}$ for the particle resolution of $\frac{l_{s}}{80}$.

radius $\frac{1}{6}[\mathrm{~m}]$ is placed at the center of a square box with length sides $\left(l_{b}=\right.$ $1[m])$. The square box contains two different fluid phases in an stratified arrangement. These fluid phases are respectively referred as fluid phases 1 (the phase at the top of the stratification) and 2 (the phase at the lower part of the stratification arrangement) whereas the lens is referred as phase 3. Due to the effect of the surface tension, the lens evolves until an equilibrium state (see figure 10). The contact angles at the triple junction point follows 
the Young's relation

$$
\frac{\sin \theta_{1}}{\sigma^{2-3}}=\frac{\sin \theta_{2}}{\sigma^{1-3}}=\frac{\sin \theta_{3}}{\sigma^{1-2}}
$$

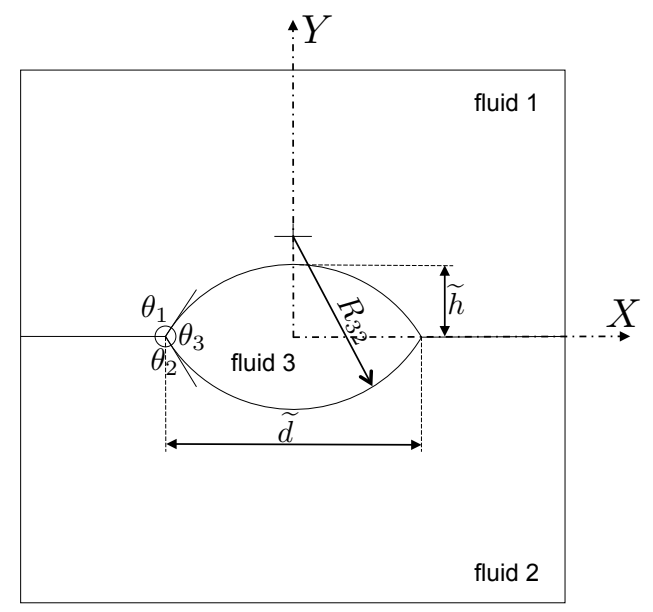

Figure 10: Schematical representation of contact angles at a triple junction point.

Fluid densities are set as $\left(\rho_{1}=\rho_{2}=\rho_{3}=1\left[\mathrm{Kg} / \mathrm{m}^{3}\right]\right)$ for the three fluid phases. The dynamic viscosities are identical for the three fluid phases $\left(\mu_{1}=\mu_{2}=\mu_{3}=0.5[\right.$ Pas.s $\left.]\right)$. The interfacial surface tension applied on all fluids interfaces is $\left(\sigma^{1-2}=\sigma^{2-3}=\sigma^{1-3}=5[N / m]\right)$, in order to obtain a symmetric lens. The analytic solution is obtained from Laplace's law and Young's relation. The theoretical value of the pressure jump between phases $(\Delta p)$ is obtained in 2D case from equation (41). The shape of the half lens ( symmetric with respect to the $X$ axis) at equilibrium state is assumed to be a circular segment with following parameters (see figure 10): The distance between the two triple junction points $(\widetilde{d})$, the contact angles of the $i^{\text {th }}$ phase $\left(\theta_{i}\right)$, the sagitta (the distance from the center of the arc to the center of its base) of the segment $(\widetilde{h})$, the radius of the curvature of the interface between the phases $i$ and $j\left(R_{i j}\right)$ (in figure 10, we represent the curvature between the upper fluid of the stratification (1) and the fluid of the lens (3) ).

$$
\Delta p=p_{i}-p_{j}=\frac{\sigma^{i-j}}{R_{i j}}
$$


At the equilibrium state the lens area $A$, the distance between triple junction points $\widetilde{d}$, and the contact angles $\theta_{i}$ can be expressed follows the relation [49]

$$
\widetilde{d}=\left(\frac{1}{8 A}\left(\frac{2\left(\pi-\theta_{1}\right)-\sin \left(\left(\pi-\theta_{1}\right)\right)}{\sin ^{2}\left(\pi-\theta_{1}\right)}+\frac{2\left(\pi-\theta_{2}\right)-\sin \left(\left(\pi-\theta_{2}\right)\right)}{\sin ^{2}\left(\pi-\theta_{2}\right)}\right)\right)^{-\frac{1}{2}} \approx 0.4617[m]
$$

Note that in this test case, the surface tension coefficients are taken identical. Then, the Young's relation (equation (40)) reads as $\theta_{1}=\theta_{2}=\theta_{3}=\frac{2 \pi}{3}$.

Geometrical consideration leads to the following results

$$
\begin{aligned}
& R_{31}=R_{32}=\frac{\widetilde{d}}{2 \sin \frac{\theta_{3}}{2}} \approx 0.2665[\mathrm{~m}] \\
& \widetilde{h}=R_{32}\left(1-\cos \frac{\theta_{3}}{2}\right) \approx 0.1333[\mathrm{~m}]
\end{aligned}
$$

All the particles are initially at rest. The no-slip boundary condition is applied on the upper and lower boundaries. On the left and right boundaries a periodic boundary condition is applied. The reference speed of sound and the background pressure are taken as $\left(\left(c_{0}=55[\mathrm{~m} / \mathrm{s}]\right)\right.$ and $\left.\left.p_{b}=150[\mathrm{~Pa}]\right)\right)$.

The time evolution of kinetic energy for three different resolutions $\frac{l_{b}}{40}, \frac{l_{b}}{80}$ and $\frac{l_{b}}{160}$ is plotted in the figure 11. The kinetic energy converges to approximate zero and reaches the equilibrium state. As it is observed, the magnitude of the velocity decreases considerably (order of $O\left(10^{-2}\right)$; see figure 12 ) at later time for the three particle resolutions. This indicates that the parasitic currents do not create a serious effect on the obtained results.

R2: Figure 12 shows the pressure field, the particle distribution of three phases stratification arrangement and magnitude of velocity filed at the equilibrium state. The initial circular shape of the lens evolved to an elliptical shape at the equilibrium state. The pressure jump profile along $X=0$ and $Y=0$ lines are plotted with the analytical solution obtained from equation (41), which gives $\Delta p=p_{3}-p_{1}=p_{3}-p_{2} \approx 18.7591[\mathrm{~Pa}]$. Quantitatively a good agreement between the numerical solution and analytical one is observed for both pressure profiles (along the $X=0$ and $Y=0$ lines). A 


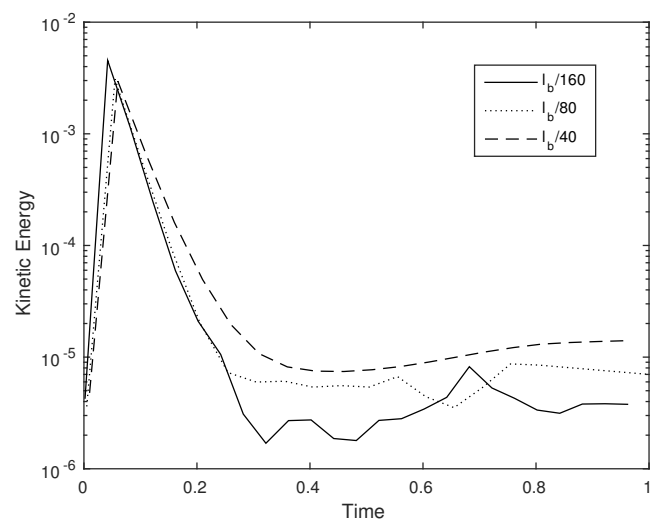

Figure 11: Spreading lens between two stratified layers: Time-evolution of kinetic energy for three different particle resolutions.

pressure instability is appeared near to the triple junctions points (following the $X$ axis) for both resolutions (see figure 13). This instability tend to disappear with increasing of resolution ( The instability in low resolution is more marked than in higher one). Note that for a higher particle resolution, the SPH numerical solution closes to the analytical one in terms of pressure and geometrical details of lens which guarantees thereafter the convergence of the proposed SPH interfacial multiphase model to deal with a triple junction points problems.
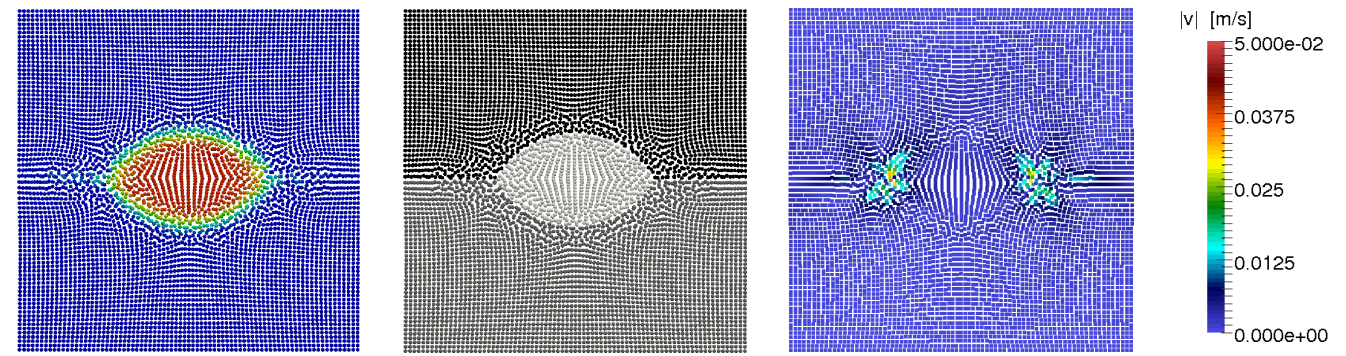

Figure 12: Spreading lens between two stratified layers: pressure field (left), particle distribution of three-phases stratification arrangement (middle) and magnitude velocity filed (right).

The wettability of a fluid phase in contact with other two phases can occur either partially or totally, depending on the surface tensions between 

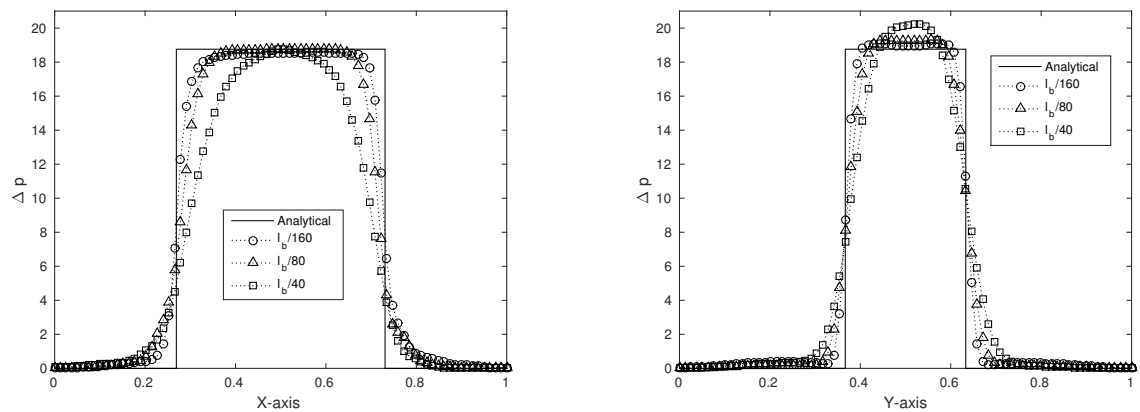

Figure 13: Spreading lens between two stratified layers: pressure jump profile along $Y=0$ (left) and $X=0$ (right) lines.

the three fluid interfaces. The degree of wettability can be determined by the spreading parameter $S_{p}$ [50]. If the spreading parameter is positive the fluid of this phase will spread completely on the interface between the other phases (total spreading). Here, in order to check if the proposed method reproduces accurately this phenomenon, we consider two different configurations following the work presented in [40].

We define the spreading parameter for the lens $\left(S_{p_{3}}\right)$ and the upper fluid $\left(S_{p_{1}}\right)$ phases as follows

$$
\begin{aligned}
& S_{p_{3}}=\sigma^{1-2}-\left(\sigma^{1-3}+\sigma^{2-3}\right) \\
& S_{p_{1}}=\sigma^{2-3}-\left(\sigma^{1-2}+\sigma^{1-3}\right)
\end{aligned}
$$

The first configuration of the problem is defined by choosing the values of interfacial surface tensions as $\left\{\sigma^{1-2}, \sigma^{1-3}, \sigma^{2-3}\right\}=\{3,1,1\}[N / m]\left(S_{p_{3}}=\right.$ $1[N / m]>0)$. This choice leads to the total spreading of the lens phase 3 on the interface phases $1-2$

For the second configuration, the values of interfacial surface tensions are chosen as $\left\{\sigma^{1-2}, \sigma^{1-3}, \sigma^{2-3}\right\}=\{1,1,3\}[N / m]\left(S_{p_{1}}=1[N / m]>0\right)$. This choice leads to the total spreading of the upper fluid phase 1 on the interface phases 2-3.

In Figure 14 we show the evolution of the spreading until the equilibrium state is reached for the two configurations considered. In both cases, the triple points disappear and the equilibrium interfaces are plane in the first 
case and sphericalsecond in the second simulation case. The results obtained are in agreement with those obtained in [40].

(a)

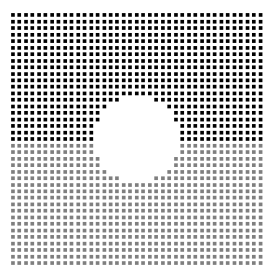

(b)

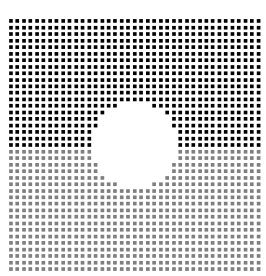

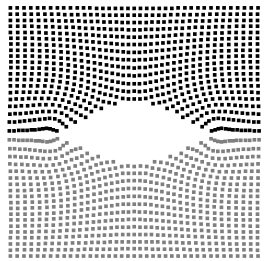

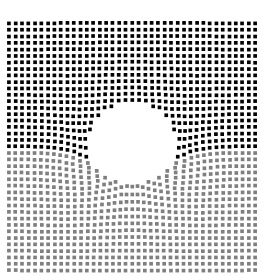

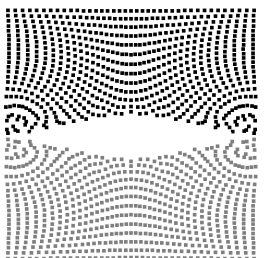
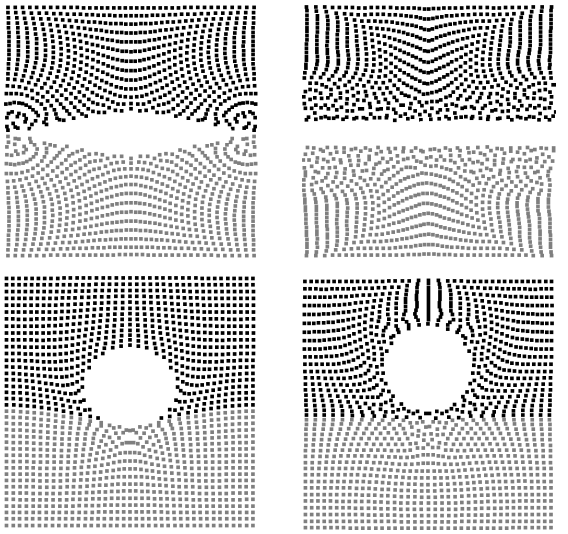

Figure 14: Total spreading evolution of the fluid lens for the configurations : a) $\left\{\sigma^{1-2}, \sigma^{1-3}, \sigma^{2-3}\right\}=\{3,1,1\}[N / m]$ and b) $\left\{\sigma^{1-2}, \sigma^{1-3}, \sigma^{2-3}\right\}=\{1,1,3\}[N / m]$.

\subsubsection{Capillary-viscous wave}

In this example, the dynamic test case involving a liquid-droplet oscillation in a liquid phase under the action of capillary forces is investigated. This test was already simulated in the SPH framework in several works such as $[14,15,16,17,2]$. The liquid-droplet (referenced with a $d$ subscript) has a radius of $R=0.1875[\mathrm{~m}]$ and is surrounded with another liquid phase (referenced with a $l$ subscript). Both fluids are placed at the center of a square box of size $L_{x}=L_{y}=1[\mathrm{~m}]$. The densities and dynamic viscosities of the droplet and its surrounding fluid are considered the same and equal to $\rho_{d}=\rho_{l}=1\left[\mathrm{Kg} / \mathrm{m}^{3}\right], \mu_{d}=\mu_{l}=0.05[\mathrm{~Pa} . \mathrm{s}]$, respectively. The surface tension coefficient between the two phases is set as unity $\left(\sigma^{d-l}=1[N / m]\right)$. A divergence-free initial velocity field is assigned to all fluid particles and it reads as

$$
\begin{gathered}
v_{x}=v_{0} \frac{x}{r_{0}}\left(1-\frac{y^{2}}{r_{0} r}\right) e^{-\frac{r}{r_{0}}} \\
v_{y}=-v_{0} \frac{y}{r_{0}}\left(1-\frac{x^{2}}{r_{0} r}\right) e^{-\frac{r}{r_{0}}}
\end{gathered}
$$


Where $r$ denotes the distance between the particle position $(x, y)$ and the droplet center. Terms $v_{0}$ and $r_{0}$ are the characteristic velocity and distance, and are taken as $v_{0}=10[\mathrm{~m} / \mathrm{s}]$ and $r_{0}=0.05[\mathrm{~m}]$. We study the convergence properties using three different number of particles 900, 3600, 14400 particles. The reference speed of sound is chosen equal to $c_{0}=10[\mathrm{~m} / \mathrm{s}]$ for both fluid phases under all resolutions. A positive background pressure is set as $\left(p_{b}=5[P a]\right)$. A no-slip boundary condition is applied on the square box sides.

Figure 15 shows droplet particles positions at $t=\{0.0,0.08,0.16,0.26\}[s]$, under the resolution of 14400 particles ( about the same resolution used by Morris et al [14] ). A good agreement is observed comparing with Morris et al [14] and also with Adami et al results (their figure 6 ). R1: As it is shown in the figure 16, the present SPH model provides more regular particle distribution and more smooth interface comparing with the results obtained by [14] with the consideration that initial distribution of the particles are different.

Figure 17 shows the time evolution of the center of mass position of the upper right-quarter section of the droplet with different resolutions. It is observed that with increasing resolution the difference in results becomes less significant (the results with resolutions 3600 and 14400 particles are very close comparing with the lowest resolution of 900 particles).

\subsection{Second category of tests: Validation of the formulation for simulation of} multiphase flows under gravity effects neglecting the surface tension

This set of tests are designed to check if the presented formulation can simulate multiphase flows under gravity effects. In these examples, the surface tension force is neglected. Before addressing the Rayleigh-Taylor instability problem, we investigate first the ability of the current SPH model to reach a hydrostatic pressure condition starting with zero initial pressures

\subsubsection{Vertical fluid column: Hydrostatic pressure condition}

In this test, we set three configurations of two stratified fluid layers which have the same dimensions $([0, L] \times[0,2 L]$ (see figure 18) with different density ratio $\frac{\rho_{2}}{\rho_{1}}=1, \frac{\rho_{2}}{\rho_{1}}=2$ and $\frac{\rho_{2}}{\rho_{1}}=4$ [51]. Each test case is investigated using three different particle resolutions $\{24 \times 49,49 \times 99,99 \times 199\}$. The subscripts 1 and 2 denote the upper and the lower fluids, respectively.

The half-length of the column is chosen as $L=1[\mathrm{~m}]$, the interface between two fluids is located at the middle of the column (at height equals to $L$ ). The 

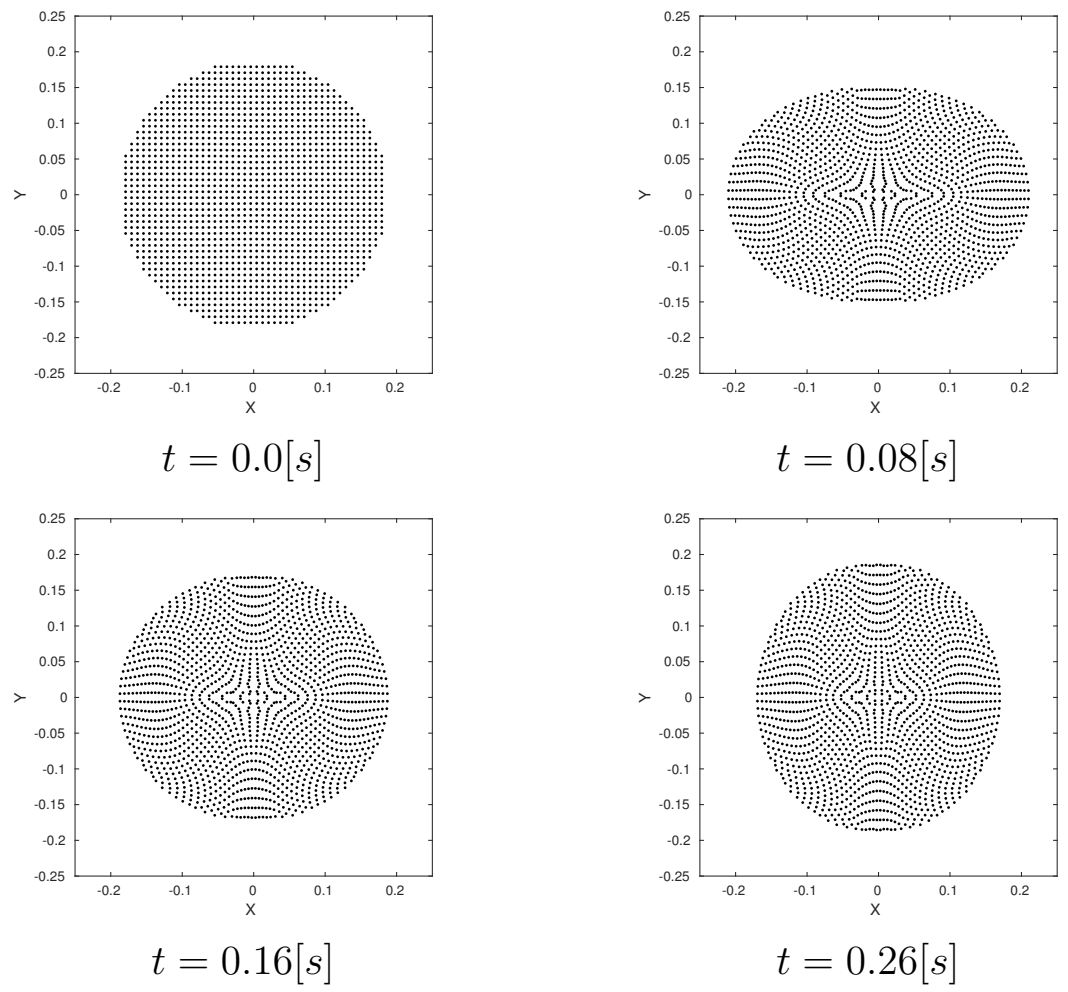

Figure 15: Droplet oscillation : droplet particle positions at $t=\{0.0,0.08,0.16,0.26\}[s]$.

initial particle distribution is a regular lattice for each of the three resolutions considered. The density of the lighter fluid is $\rho_{1}=1\left[\mathrm{~kg} / \mathrm{m}^{3}\right]$. The dynamic viscosity is chosen constant as $\mu_{1}=\mu_{2}=0.1$ [Pas.s] for all phases in all the configurations. The vertical fluid column is assumed to be under the action of an unit gravity $\left(\mathbf{g}=(0,-1)\left[\mathrm{m} / \mathrm{s}^{2}\right]\right)$. The reference speeds of sound are chosen according to the condition discussed in Section 2.2, resulting in the values of $c_{01}=10[\mathrm{~m} / \mathrm{s}]$ for all configurations and $c_{02}=\{20,14.142,10\}[\mathrm{m} / \mathrm{s}]$ for the configurations of $\frac{\rho_{2}}{\rho_{1}}=1, \frac{\rho_{2}}{\rho_{1}}=2$ and $\frac{\rho_{2}}{\rho_{1}}=4$, respectively. The background pressure is chosen as $\left(p_{b}=0.05 p_{r}=5[\mathrm{~Pa}]\right)$. The no-slip boundary condition is applied at all boundaries.

In order to show the efficiency of the damping technique to reach quickly the equilibrium state, the three configurations are tested with and without the damping technique for the coarsest particle resolution $24 \times 49$. The value of the damping period is chosen as $T_{D}=1[s]$. 

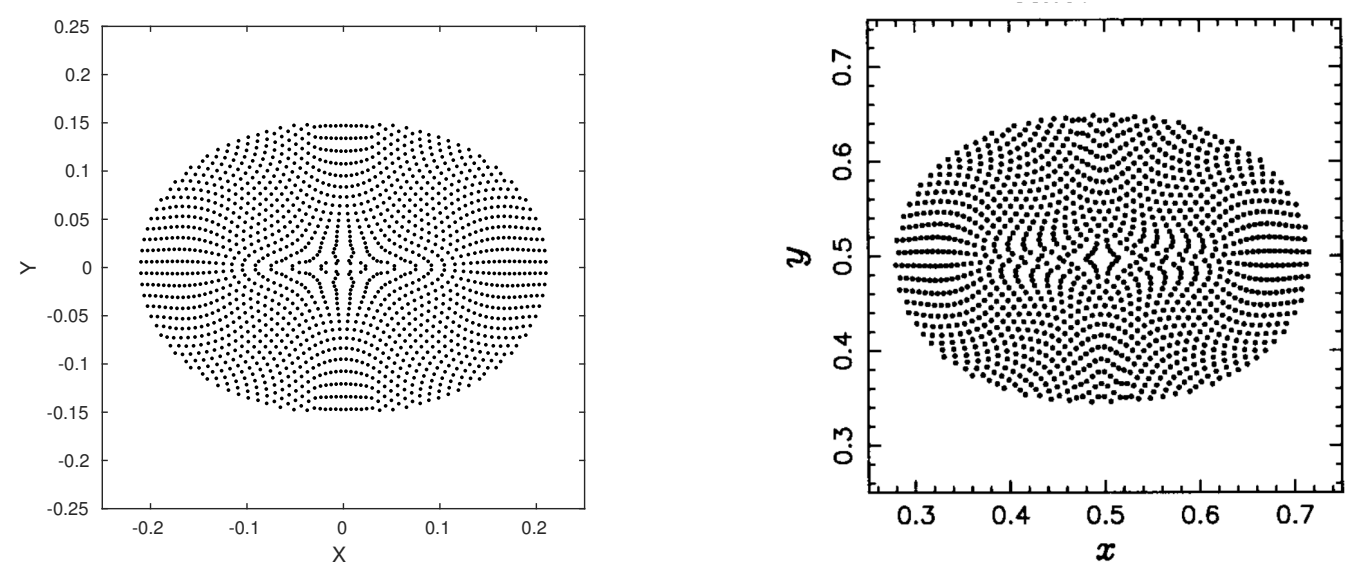

$$
t=0.08[s]
$$
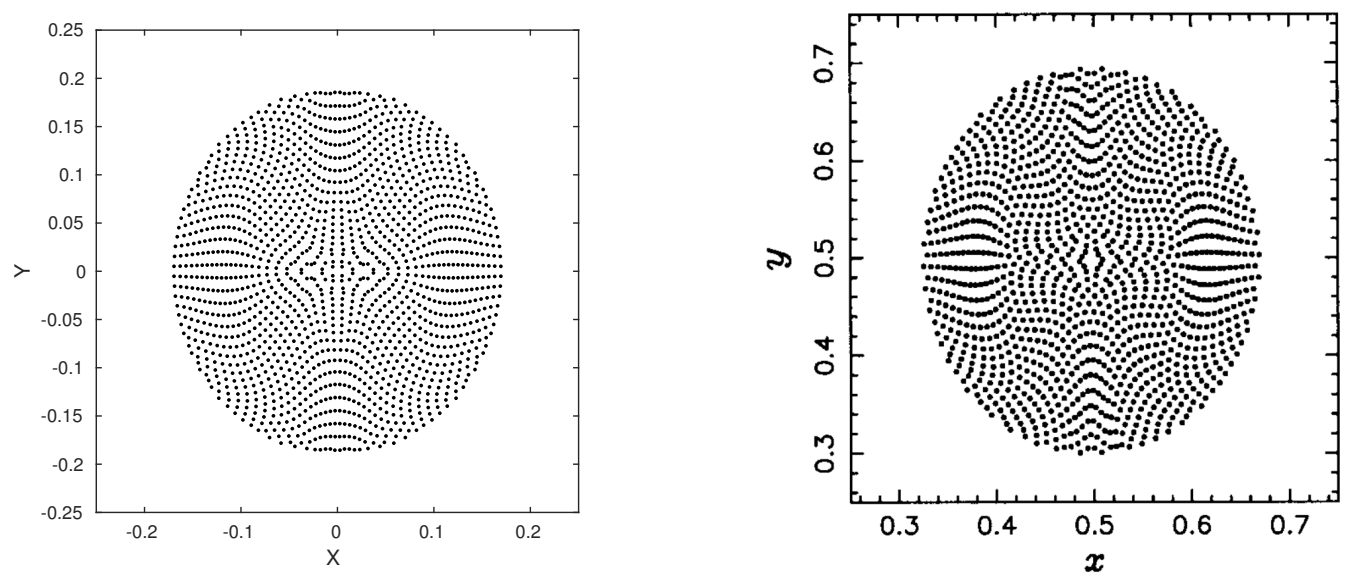

$$
t=0.26[s]
$$

Figure 16: R1: Comparison between the particles distribution of the oscillating droplet at $t=0.08[s]$ and $t=0.26[s]$ under the resolution of 14400 particles. The left column presents our results. The right column presents the resukts of Morris [14].

Figure 19 shows the effect of the damping technique on the simulation results for the pressure for the three configurations. We can see clearly that using the damping technique leads to lower amplitude of the pressure oscillations and to a faster convergence to the stabilized hydrostatic value. It is also observed that for the highest density ratio, the pressure has an important amplitude of oscillation.In this case, using the damping technique reduces the amplitude of the oscillations, but they are still important. This is principally due to 


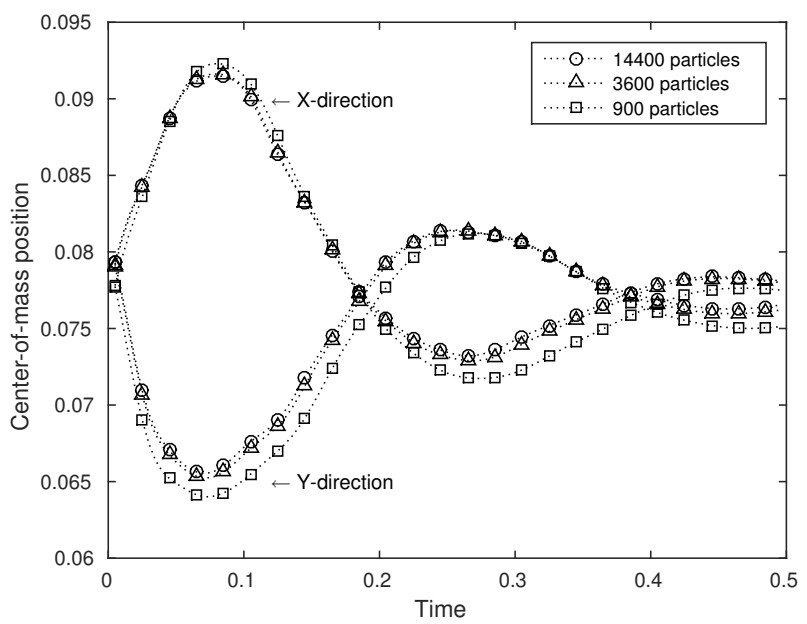

Figure 17: Convergence test of droplet oscillation: Center of mass position of the upper right-quarter section of the droplet.
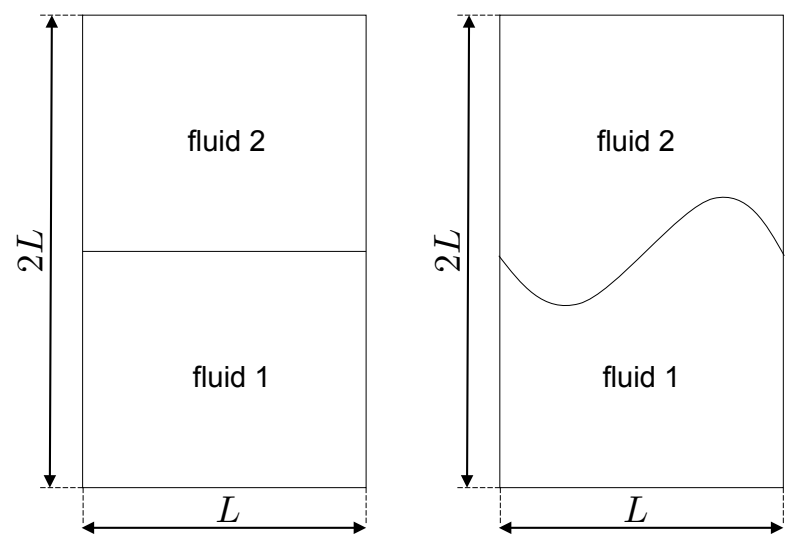

Figure 18: Geometrical details of the initial configuration of vertical fluid column: The figure on the left describes the setup of the problem for the hydrostatic pressure condition. On the right, we show the initial state for the Rayleigh-Taylor instability test.

the change of the physical parameters of the lighter fluid after the damping time. This change creates the jump in density, viscosity and mass and thus the pressure. Despite that, it converges faster and presents less oscillations than in the case of the simulation without using of the damping technique. A remedy to further reduce the oscillations is to increase the damping time $\left(T_{D}\right)$. In the case of unit density ratio the oscillations of the pressure are 
very limited and reachs the stabilized value of the hydrostatic pressure very quickly.
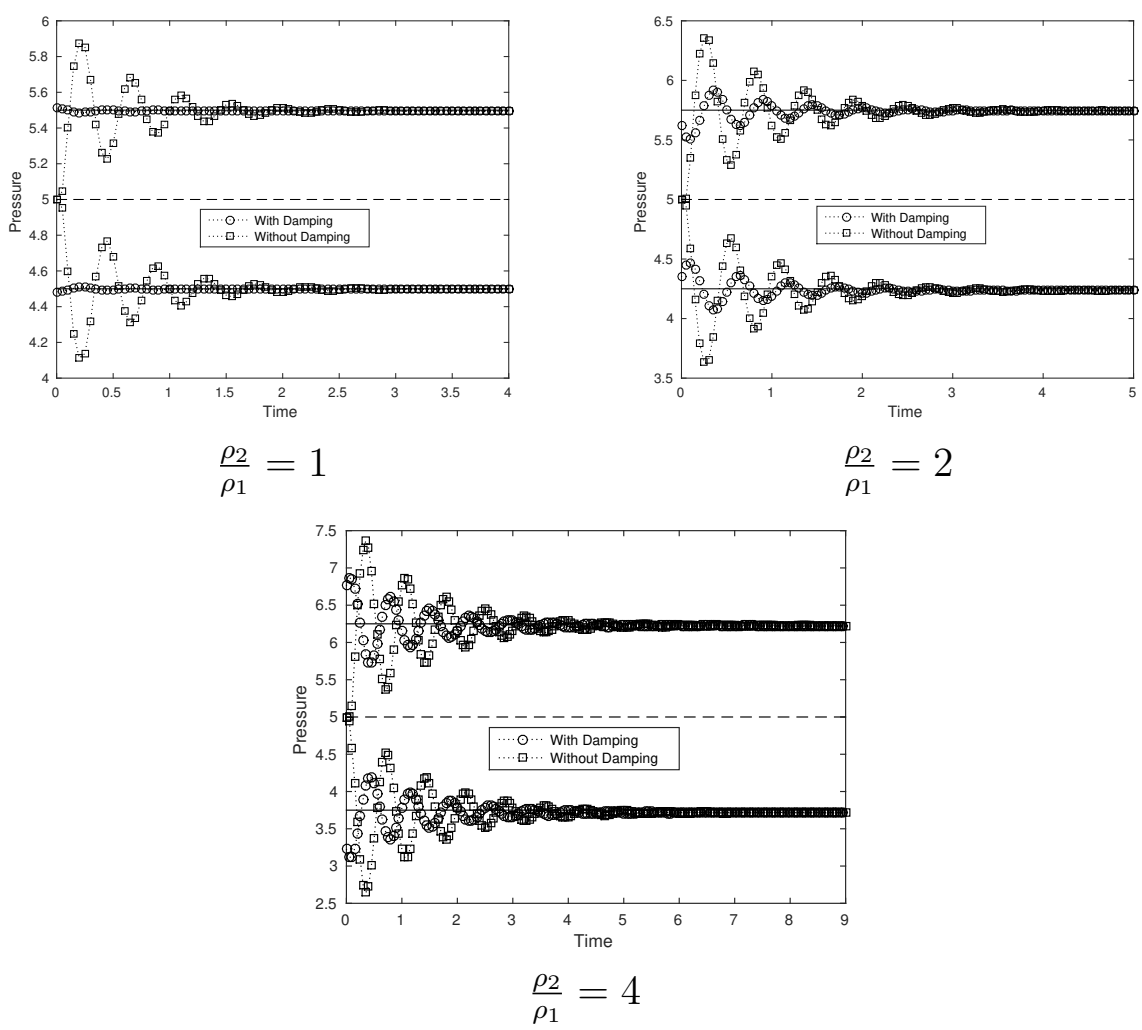

Figure 19: Time-evolution of pressure at the point $(0.5,0.5)$ (upper curves) and $(0.5,1.5)$ (lower curves) with (Dotted line with small circle) and without (dotted line with small square) the damping technique. The solid lines represent the stabilized pressure, and the dashed line presents the background pressure.

After the stabilization of the pressure field using the damping technique, we compare the obtained pressure profiles using the present SPH model with the analytical ones for different density ratios and different particle resolutions (see figure 20). The numerical results agree well with the analytical ones.

In figure 21, we show the particle distribution and hydrostatic pressure at equilibrium for the three particle resolutions for the $\frac{\rho_{2}}{\rho_{1}}=4$ case. Pressure isolines are plotted in order to check the hydrostatic pressure levels obtained with the tree different resolutions. It is observed that the same pressure levels are reached for all the particle resolutions. This indicates the convergence of 
the presented numerical model for the simulation of gravitational multiphase fluid flows. Note that the background pressure $p_{b}=5[\mathrm{~Pa}]$ is included in the range of computed pressure variation.

From the results presented in figures 20 and 21 we can also conclude that the proposed modifications to the generalized wall boundary conditions method [3] (see section 2.3) give good results. In order to perform an additional analysis of the efficiency of this method, the same example was investigated under high density and viscosity ratios. The density ratio was chosen to be equal $\frac{\rho_{2}}{\rho_{1}}=100$, with $\rho_{2}=100\left[\mathrm{~kg} / \mathrm{m}^{3}\right]$ and $\rho_{1}=1\left[\mathrm{~kg} / \mathrm{m}^{3}\right]$. While the viscosity ratio is taken as $\frac{\mu_{2}}{\mu_{1}}=10$, with $\mu_{1}=0.01[$ Pa.s $]$ and $\mu_{2}=0.1[\mathrm{~Pa} . s]$. The reference R1: speed of sound of the fluid phase 2 is set to $c_{0_{2}}=10[\mathrm{~m} / \mathrm{s}]$ and $c_{0_{1}}=100[\mathrm{~m} / \mathrm{s}]$ for the phase 1 that give a reference pressure of $p_{r}=10^{4}[\mathrm{~Pa}]$. The background pressure is taken as $p_{b}=0.05 p_{r}=500[P a]$. The simulation was performed using $49 \times 99$ particles, a damping period of $T_{D}=1[s]$ and no-slip condition is applied on all wall boundaries.

After the damping period $\left(T_{D}=1[s]\right)$ the hydrostatic pressure field oscillates until it reaches the stabilized values at time $t=18[s]$ as it is shown in the figure 22. When the pressure field stabilizes, we perform a comparison between the numerical and analytical hydrostatic pressure profiles taken from the centerline of the vertical fluid column. The results are shown in figure 23. A very satisfactory agreement is observed between the pressure results obtained with the present approach and analytical results. Figure 24 shows the vertical fluid column particles distribution at stabilized state. The fluid particles are colored with phases (gray for the fluid 2 and black for the fluid 1) in order to show that the interface between the two fluid phases that meet the vertical wall boundaries is stable. As it is shown in the center of the figure 24, there are spurious currents which present a maximum velocity magnitude of order $O\left(10^{-3}\right)$. A smooth hydrostatic pressure field is observed via the right side of figure 24 .

\subsubsection{Rayleigh-Taylor instability}

The Rayleigh-Taylor instability (RTI) is a widely used benchmark [52, 32, $18,41]$ to test the accuracy of numerical methods for multiphase gravity flows. The Rayleigh-Taylor instability, occurs at an interface between two fluids of different densities when the lighter fluid pushes the heavier fluid. 
This phenomenon occurs in a multitude of physical (salt domes, weather inversions, etc) and industrial applications.

In this work a Rayleigh-Taylor with sinusoidal asymmetric interface perturbation is studied. The computational domain is $[0, L] \times[0,2 L]$ with $L=1[\mathrm{~m}]$. The computational domain is divided in two sub-domains by a sinusoidal interface $y(x)=1-0.15 \sin (2 \pi x)$ (see figure 18). The lower sub-domain is occupied by the lighter fluid (referred as phase 1) which has a density $\rho_{1}=1\left[\mathrm{~kg} / \mathrm{m}^{3}\right]$, while the upper sub-domain is occupied by the heavier fluid (referred as phase 2) with density $\rho_{2}=1.8\left[\mathrm{~kg} / \mathrm{m}^{3}\right]$. The Reynolds number is defined here as $R_{e}=\frac{\sqrt{\|\mathbf{g}\| L^{3}}}{\nu}$, and a value of $R_{e}=420$ is chosen. In the previous definition, $\|\mathbf{g}\|=1\left[\mathrm{~m} / \mathrm{s}^{2}\right]$ is the modulus of the gravity acceleration vector, and $\nu=\nu_{1}=\nu_{2}=0.0024\left[\mathrm{~m}^{2} / \mathrm{s}\right]$ is the kinematic viscosity which is chosen to be equal and constant for both fluids phases.

The particles have an initial regular lattice distribution. The RTI test is solved with three different particles resolutions $\{49 \times 99,99 \times 199,133 \times 267\}$ . The reference speeds of sound are taken as $\left\{c_{01}, c_{02}\right\}=\{13.41,10\}[\mathrm{m} / \mathrm{s}]$, for the lighter and heavier fluids, respectively. The reference pressure is chosen equal to $9[\mathrm{~Pa}]$. The no-slip boundary condition is applied on all solid boundaries.

In figure 25 we compare three different particle resolutions at three different times $t=1[s], t=3[s], t=5[s]$. Quantitatively, it's shown that the three particle resolutions are able to simulate substantially the same phenomena of RTI. Nevertheless, at the low resolution $49 \times 99$ the roll-up of the small structures at the mushroom-shaped head are not well reproduced. For the finer resolutions, all the small structure phenomena due to the development and roll-up of the mushroom-shaped heads are captured. The two finer resolutions $(99 \times 199$ and $150 \times 300)$ are very similar to each other in terms of the shape of the instability.

We also performed a comparison between the RTI interfaces reproduced with the proposed SPH model and two different numerical methods. In figure 26, we compare the results at time $t=5[s]$ with the resolution of $150 \times 300$ particles with those of a Level-set method [18] and another SPH model [41]. There are some differences between the SPH method [41] and the method presented here. For instance in [41] a Wendland kernel function [53] and time-integration of continuity equation for the density are used instead of the quintic spline kernel 7 and summation-based density 8 used in the presented 
SPH method, respectively. The present SPH model can reproduce the RTI interface in good agreement with the other numerical models which have more resolution (Level-Set: a grid of $312 \times 624$ cells, and SPH model[41]:150 $\times 300$ particles). Globally, the RTI interface reproduced with the proposed SPH approach is closer to that reproduced with the SPH model of [41]. This is probably due to the same nature of both models. We note, however that in some places the interface with the proposed SPH method is closer to that obtained by the Level-set one [18]. These results are only of a qualitative nature. Monaghan et al [41] prefer to examine the convergence by comparing the time evolution of the position of the $Y$-coordinate of the highest particle of the lighter fluid (phase 1) with that obtained from the Layzer's theory $[42,54]$. Thus, the highest point of the phase 1 fluid for the three particle resolutions $\{49 \times 99,99 \times 199,150 \times 300\}$ is plotted in figure 27 together with the curve obtained using the Layzer's theory. The results are in good agreement with the Layzer theory. Note that the the Layzer theory is for a periodic domain, while the results are obtained from the simulation in a rectangular rigid domain with no-slip boundaries. This fact may explain some of the deviations of the numerical results from the theoretical line.

\subsection{Third category of tests: high density and viscosity ratio effects}

The simulations of the bubble rising behaviour can be characterized by the Reynolds and the Eötvös dimensionless numbers [43] and also the density and viscosity ratio $\left(\frac{\rho_{1}}{\rho_{2}}, \frac{\mu_{1}}{\mu_{2}}\right)$. The Reynolds number $R_{e}$ gives the ratio of inertial to viscous effects and is expressed as

$$
R_{e}=\frac{\rho_{1} v_{c} L_{c}}{\mu_{1}}
$$

While the Eötvös number $E_{o}$ compares buoyancy effects to capillary ones :

$$
E_{o}=\frac{\rho_{1} v_{c}^{2} L_{c}}{\sigma}
$$

In this section, the subscripts 1 and 2 refer to the heavier and lighter fluid, respectively. The characteristic velocity is defined as $v_{c}=\sqrt{2 R\|\mathbf{g}\|}$ and $L_{c}=2 R$ refers to the characteristic length. $R$ is initial radius of the bubble and $\|\mathbf{g}\|$ is the magnitude of the gravity acceleration vector. 


\subsubsection{Single bubble rising through a vertical column of water}

The purpose of this test is to show the efficiency of the present SPH model to simulate a two phase interfacial fluid flow with low and high density ratios. These tests are taken from the work of Hysing et al [43]. A single bubble rising in a vertical column due to gravity effect. The initial bubble position and dimensions of the vertical column are described related to the bubble radius $R$ R1: (see figure 28). The physical variables and parameters are summarized at Table 1 as Case 1.

This test is done with two different particle resolutions $66 \times 133$ and $133 \times 267$. The damping technique is applied here with $T_{D}=1$. The reference speeds of sound for the two phases are set to $\left\{c_{0_{1}}, c_{0_{2}}\right\}=\{5.7,18\}[\mathrm{m} / \mathrm{s}]$. No-slip boundary conditions are applied on the upper and lower solid boundaries, while free-slip boundary conditions are applied on the left and right boundaries.

The position of the gravity center $G C_{y}$ of the bubble and its vertical velocity $v_{G C}$ are computed as follows

$$
\begin{aligned}
G C_{y} & =\frac{\sum i^{N_{b}} y_{i}}{N_{b}} . \\
v_{G C} & =\frac{\sum i^{N_{b}} v_{y i}}{N_{b}} .
\end{aligned}
$$

$N_{b}$ denotes the number of particles in the bubble, and $y_{i}$ is the $Y$ coordinate of the particle $i$ which belongs to the particle set of the bubble. The term $v_{y i}$ denotes the vertical component of the velocity of the particle $i$.

In figure 29 we compare our results for the time evolution of the vertical position of the gravity center of the rising bubble and its vertical velocity with the ones obtained in [43] using the VOF method. Basically, a good agreement is found between our SPH numerical results and those of reference [43], even tough a slight difference for the position of the gravity center is detected, and some oscillations are observed around the VOF velocity curve. However, the overall SPH results are in good agreement with the reference ones.

In figure 30, we also compare the shape and position of the bubble interfaces at time $t=3[s]$. We observe a perfect agreement in this case.

Figure 31 shows the pressure and the magnitude velocity of the water column for two different particle resolutions at two different times $t=1[s]$ 
and $t=2[s]$. Moreover, in figure 32 we show the direction of the velocity vector of the particles inside the bubble for the previous two resolutions at time $t=1[s]$. The results obtained for both particle resolutions are very similar, indicating the convergence of the numerical model.

Now we address the same case with a higher density ratio [44, 28, 18]. In this case, during the rising motion, the bubble undergoes a large deformation that subsequently splits it into three parts. The initial setup is presented in figure 18 and the physical variables and parameters for this test case are summarized at Table 1 as the Case 2.

In this test we use a discretization of $240 \times 400$ particles. The reference speeds of sound are $\left\{c_{0_{1}}, c_{0_{2}}\right\}=\{7,221.35\}[\mathrm{m} / \mathrm{s}]$. The damping technique is used here with $\left(T_{D}=0.05[s]\right)$. The boundary conditions are the same than for Case 1 of this section.

Figure 33 shows the velocity and relative pressure $\left(\widetilde{\Delta p}=p-p_{\min }\right)$ of the column of water at the dimensionless time $t \sqrt{\|\mathbf{g}\| / R}=3.6$. The bubble is strongly deformed and it is split in three parts during its evolution. The evolution of the bubble is presented in figure 34 during nine different instants. The particles inside the bubble are colored with the magnitude of the velocity. In figure 35 we compare the results obtained with the SPH method with those obtained using a Level-Set method [44]. The results of the SPH and LevelSet methods are in good agreement. During the rising process, the bubble deforms and takes a horseshoe shape. After that, the extremities roll-up until they undergo a big deformation which subsequently splits the bubble to form other small ones. The main difference between the results is that near to the symmetric axis the bubble obtained using the present SPH method is thicker than the one obtained by the level-Set method. The results for the width of the bubble remains in very good agreement during all the simulation period for both methods. We observe that in the Level-set solution the bubble splits in several very small bubbles that are not predicted by the present SPH method. Thereafter, these smaller bubbles disappear gradually from the Level-Set simulation.

\begin{tabular}{|c|c|c|c|c|c|c|c|c|c|c|}
\hline Case & $\rho_{1}\left[\mathrm{~kg} / \mathrm{m}^{3}\right]$ & $\rho_{2}\left[\mathrm{~kg} / \mathrm{m}^{3}\right]$ & $\mu_{1}[$ Pa.s $]$ & $\mu_{2}[$ Pa.s $]$ & $\sigma[\mathrm{N} / \mathrm{m}]$ & $\|\mathbf{g}\|\left[\mathrm{m} / \mathrm{s}^{2}\right]$ & $R_{e}$ & $E_{o}$ & $\frac{\rho_{1}}{\rho_{2}}$ & $\frac{\mu_{1}}{\mu_{2}}$ \\
\hline 1 & 1000 & 100 & 10 & 1 & 24.5 & 0.98 & 35 & 10 & 10 & 10 \\
\hline 2 & 1000 & 1 & 0.035 & 0.0045 & 0.1226 & 9.81 & 1000 & 285.63 & 1000 & 7.77 \\
\hline
\end{tabular}

Table 1: Setup for Case 1 and Case 2 tests. 


\subsubsection{Gas bubble rising through two stratified fluid layers}

This test example describes the behavior of a rising gas bubble through two stratified fluid layers (see figure 36 ). The gas bubble can cross the interface between layers with or without entrainment of the heavier fluid into the lighter, or it could even remain trapped in it. Greene et al $[55,56]$ suggest a criterion on the bubble volume to predict this behavior based on a macroscopic balance between surface tension forces and buoyancy forces. Thus, if the bubble volume is greater than a critical volume $V_{c}\left(V_{b}>V_{c}\right)$, the bubble will penetrate the interface layer and it will eventually entrain into the heavier fluid, otherwise the bubble will be trapped between the interface layers. The critical volume is calculated as follows

$$
V_{c}=\left(\frac{2 \pi\left(\frac{3}{4 \pi}\right)^{1 / 3} \sigma^{2-3}}{\left(\rho_{3}-\rho_{1}\right)\|\mathbf{g}\|}\right)^{\frac{3}{2}}
$$

In equation (53) the subscript(or superscript for surface tension) 1 correspond to the lower fluid, 2 refers to the bubble, and 3 refers to the upper fluid.

This problem is very challenging from the numerical point of view since it involves high denisites and viscosities ratios and the presence of triple point junctions. The setup of the problem presented here is taken from [4]. The density and dynamic viscosity of fluid 1 (95\% glycerin + water) are $\rho_{1}=1244\left[\mathrm{~kg} / \mathrm{m}^{3}\right], \mu_{1}=550.1 \times 10^{-3}[\mathrm{Pa.s}]$, in the case of the buble (fluid 2) the chosen values are those of the air: $\rho_{2}=1.205\left[\mathrm{~kg} / \mathrm{m}^{3}\right]$ and $\mu_{2}=5 \times 10^{-3}[$ Pa.s $]$. Note that the value of air bubble viscosity is chosen greater than the real one and equal $\mu_{2}=5 \times 10^{-3}[$ Pa.s $]$ instead the use of the real gas viscosity with an artificial one to guarantee the stability of the algorithm [35]. Finally, for the fluid 3 (47V500 oil), the values are $\rho_{3}=965$. and $\mu_{3}=530.7 \times 10^{-3}[P a . s]$. The surface tensions are $\sigma^{1-2}=$ $45.1613 \times 10^{-3}[\mathrm{~N} / \mathrm{m}], \sigma^{1-3}=21 \times 10^{-3}[\mathrm{~N} / \mathrm{m}], \sigma^{2-3}=28 \times 10^{-3}[\mathrm{~N} / \mathrm{m}]$, and the speeds of sound are taken as $\left\{c_{01}, c_{02}, c_{03}\right\}=\{3.7,118.88,4.2\}[\mathrm{m} / \mathrm{s}]$. The radius of the air bubble is $R=3.5 \times 10^{-3}[\mathrm{~m}]$. These data correspond to a Reynolds number $R_{e} \approx 4.15$ and Eötvös number $E_{o} \approx 13.24$. For the simulation, a regular lattice with $50 \times 166$ particles is employed. No-slip boundary conditions are applied on the top and bottom boundaries, and periodic boundary conditions are applied on the left and right edges of the domain. The dimensions of computational domain are detailed in figure 36. The damping technique is used with $T_{D}=0.05[s]$. 
The critical volume is computed using equation (53) which gives $V_{c} \approx 3.92 \times$ $10^{-8}\left[\mathrm{~m}^{3}\right]$. This implies a critical radius of $R_{c} \approx 0.021[\mathrm{~m}]$. In this example, the initial radius of air bubble $R=0.0035[\mathrm{~m}]$ is greater than the critical one which logically involves that the $V_{c}<V_{\text {Bubble. }}$ With this values, the air bubble penetrates the interface fluid layers [4].

In figure 37 we show the numerical results for the relative hydrostatic pressure field $\widetilde{\Delta p}=p-p_{\text {min }}$, the magnitude of the velocity field and the distribution of the three different phases in different instants. The dimensionless interval between two images is taken equal to $\Delta t \sqrt{\|\mathbf{g}\| / R}=4.3$ [4]. In figure 38 we compare the numerical results with the experimental images obtained in [4]. The numerical results are in good agreement with the experimental ones. The differences between the numerical and experimental sequences are maybe due to uncertainties in the initial conditions of the experimental test and possibly to any $3 \mathrm{D}$ effect.

\subsubsection{Interaction of two rising bubbles through a fluid column}

In this last test case we solve a case based on the one presented in [57]. Two bubbles are initially set close to each other and rise through a fluid column. The upper bubble is larger than the lower one The smaller bubble has a radius $R=0.1[\mathrm{~m}]$. The geometrical setup of the problem is based on the radius of the smaller bubble and is detailed in figure 39. The physical parameters for the setup of this problem are given in Table 2 , respectively.

\begin{tabular}{|c|c|c|c|c|c|c|c|c|c|}
\hline$\rho_{1}\left[\mathrm{~kg} / \mathrm{m}^{3}\right]$ & $\rho_{2}\left[\mathrm{~kg} / \mathrm{m}^{3}\right]$ & $\mu_{1}[$ Pa.s $]$ & $\mu_{2}[$ Pa.s $]$ & $\sigma[\mathrm{N} / \mathrm{m}]$ & $\|\mathbf{g}\|\left[\mathrm{m} / \mathrm{s}^{2}\right]$ & $R_{e}$ & $E_{o}$ & $\frac{\rho_{1}}{\rho_{2}}$ & $\frac{\mu_{1}}{\mu_{2}}$ \\
\hline 1000 & 100 & 0.156 & 0.078 & 0 & 2.9 & 1794 & $\infty$ & 10 & 2 \\
\hline
\end{tabular}

Table 2: Setup for the interaction of two rising bubbles through a fluid column test case.

We use here a discretization of $500 \times 750$ particles.

The reference speeds of sound $\left\{c_{01}, c_{02}\right\}=\{9.32,29,47\}[\mathrm{m} / \mathrm{s}]$ are chosen for fluid water column and the fluid of the two bubbles, respectively. The simulation is damped for a period of $T_{D}=0.25[\mathrm{~s}]$. Left and right boundaries are set as free-Slip boundaries, whereas no-slip boundary conditions are set at top and bottom boundaries. The results for pressure variation $\widetilde{\Delta p}=p-p_{\text {min }}$ , velocity magnitude of the fluid column and of the bubble are presented in figures 40 and 41.

During the evolution process of the two rising bubbles, the upper bubble covers the lower one. This generates more efforts over the lower bubble. Due 
to this forces, the lower bubble deforms. This deformation generates two fluid ejections that go thinner as the rising process continues. Finally, the two bubbles merge but this state is not stable and they split in two parts.

\section{Conclusion}

In this work, a consistent smoothed particle hydrodynamics model is proposed. This model includes a surface tension formulation which ensures the tangential R1: properties of the tensor surface stress and the stability of the numerical method. The numerical stability is obtained through the use of a divergence operator with first order consistency and also with a damping technique that avoids the numerical issues due to the jump initial conditions. In this work it is also presented a modification of the generalized wall boundary conditions to be able to simulate multiphase fluid flows.

The accuracy, stability and applicability of the proposed SPH model to deal with interfacial multiphase problems were shown. The proposed SPH scheme is able to obtain very accurate results when dealing with problems including high density and viscosity ratios and triple junction points.

\section{Acknowledgments}

X. Nogueira and L. Ramirez have been partially supported by the Ministerio de Economía y Competitividad (grant \#DPI2015-68431-R) of the Spanish Government and by the, Consellería de Cultura, Educación e Ordenación Universitaria of the Xunta de Galicia (grant \#GRC2014/039) cofinanced with FEDER funds, and the Universidade da Coruña.

\section{Appendix A}

In this Appendix we show the relation between CSS and CSF formulations. The Continuum Surface Stress (CSS) formulation reads

$$
\mathbf{F}^{S T}=\nabla . \Pi
$$

Replacing the stress tensor $\boldsymbol{\Pi}$ by its formulation in the equation (54) we get: 


$$
\mathbf{F}^{S T}=\nabla \cdot(\sigma \underbrace{\|\nabla C\| \mathbf{I}}_{(I)}-\sigma \overbrace{\frac{\nabla C}{\|\nabla C\|} \otimes \nabla C}^{(I I)})
$$

The divergence of the first part $(I)$ of the equation (55) can be modified as

$$
\nabla \cdot(\|\nabla C\| \mathbf{I})=\nabla(\|\nabla C\|)=\left(\frac{\nabla C}{\|\nabla C\|} \cdot \nabla\right) \nabla C .
$$

Whereas the divergence of the second part $(I I)$ can be written as

$$
\nabla \cdot\left(\frac{\nabla C}{\|\nabla C\|} \otimes \nabla C\right)=\nabla \cdot\left(\frac{\nabla C}{\|\nabla C\|}\right) \nabla C+\left(\frac{\nabla C}{\|\nabla C\|} \cdot \nabla\right) \nabla C .
$$

By subtracting the equation (57) from equation (56), we obtain the Stress Surface Force formulation (CSF) (58):

$$
\mathbf{F}^{S T}=-\sigma \nabla \cdot\left(\frac{\nabla C}{\|\nabla C\|}\right) \nabla C=\sigma \kappa \nabla C .
$$

Where $\kappa$ describes the interface curvature which is expressed as $\kappa=-\nabla \cdot \mathbf{n}=$ $-\nabla \cdot\left(\frac{\nabla C}{\|\nabla C\|}\right)$, with .n is the unit normal vector to the interface.

\section{Appendix B}

In this appendix, we show that the stress surface tension is tangential to the fluid interface. We can write the surface tension as a volumetric force as follows:

$$
\mathbf{F}^{S T}=\nabla \cdot \Pi
$$

Where $\boldsymbol{\Pi}=\left(\sigma\|\nabla C\| \mathbf{I}-\sigma \frac{\nabla C}{\|\nabla C\|} \otimes \nabla C\right)$ is the stress surface tensor.

This formulation allows the interpretation of the tension force as a internal body forces of the continuum medium (as viscous tensor). So, we will investigate if these efforts are carried by the tangent direction to the interface. 
For this reason we will applied the stress tensor $\boldsymbol{\Pi}$ on an arbitrary vector $\mathbf{f}$ see figure 42 :

$$
\begin{gathered}
\boldsymbol{\Pi} \cdot \mathbf{f}=\sigma\|\nabla C\|(\mathbf{f}-(\mathbf{f} . \mathbf{n}) \mathbf{n}) \\
\mathbf{\Pi} \cdot \mathbf{f}=\sigma\|\nabla C\| \Xi_{T} \cdot \mathbf{f}
\end{gathered}
$$

Where $\Xi_{T}$ is the projection operator on the tangent plane to the interface. Equation (61) indicates that the internal forces associated to the surface stress tensor are tangent to the interface.

\section{Appendix $\mathrm{C}$}

In this appendix, we summarize the proposed SPH model via a pseudocode.

\section{Bibliography}

[1] J. Brackbill, D. B. Kothe, and C. Zemach, "A continuum method for modeling surface tension," Journal of computational physics, vol. 100, no. 2, pp. 335-354, 1992.

[2] S. Adami, X. Hu, and N. Adams, "A new surface-tension formulation for multi-phase sph using a reproducing divergence approximation," Journal of Computational Physics, vol. 229, no. 13, pp. 5011-5021, 2010.

[3] S. Adami, X. Hu, and N. Adams, "A generalized wall boundary condition for smoothed particle hydrodynamics," Journal of Computational Physics, vol. 231, no. 21, pp. 7057-7075, 2012.

[4] R. Bonhomme, J. Magnaudet, F. Duval, and B. Piar, "Inertial dynamics of air bubbles crossing a horizontal fluid-fluid interface," Journal of Fluid Mechanics, vol. 707, pp. 405-443, 2012.

[5] C. W. Hirt and B. D. Nichols, "Volume of fluid (vof) method for the dynamics of free boundaries," Journal of computational physics, vol. 39, no. 1, pp. 201-225, 1981. 
[6] S. Osher and R. P. Fedkiw, "Level set methods: an overview and some recent results," Journal of Computational physics, vol. 169, no. 2, pp. 463502, 2001.

[7] G. Tryggvason, B. Bunner, A. Esmaeeli, D. Juric, N. Al-Rawahi, W. Tauber, J. Han, S. Nas, and Y.-J. Jan, "A front-tracking method for the computations of multiphase flow," Journal of Computational Physics, vol. 169, no. 2, pp. 708-759, 2001.

[8] S. O. Unverdi and G. Tryggvason, "A front-tracking method for viscous, incompressible, multi-fluid flows," Journal of computational physics, vol. 100, no. 1, pp. 25-37, 1992.

[9] M. J. Berger and P. Colella, "Local adaptive mesh refinement for shock hydrodynamics," Journal of computational Physics, vol. 82, no. 1, pp. 64-84, 1989.

[10] R. A. Gingold and J. J. Monaghan, "Smoothed particle hydrodynamics: theory and application to non-spherical stars," Monthly notices of the royal astronomical society, vol. 181, no. 3, pp. 375-389, 1977.

[11] L. B. Lucy, "A numerical approach to the testing of the fission hypothesis," The astronomical journal, vol. 82, pp. 1013-1024, 1977.

[12] J. J. Monaghan, "An introduction to sph," Computer physics communications, vol. 48, no. 1, pp. 89-96, 1988.

[13] B. Lafaurie, C. Nardone, R. Scardovelli, S. Zaleski, and G. Zanetti, "Modelling merging and fragmentation in multiphase flows with surfer," Journal of Computational Physics, vol. 113, no. 1, pp. 134-147, 1994.

[14] J. P. Morris, "Simulating surface tension with smoothed particle hydrodynamics," International journal for numerical methods in fluids, vol. 33, no. 3, pp. 333-353, 2000.

[15] X. Y. Hu and N. A. Adams, "A multi-phase sph method for macroscopic and mesoscopic flows," Journal of Computational Physics, vol. 213, no. 2, pp. 844-861, 2006.

[16] X. Hu and N. A. Adams, "An incompressible multi-phase sph method," Journal of computational physics, vol. 227, no. 1, pp. 264-278, 2007. 
[17] X. Hu and N. Adams, "A constant-density approach for incompressible multi-phase sph," Journal of Computational Physics, vol. 228, no. 6, pp. 2082-2091, 2009.

[18] N. Grenier, M. Antuono, A. Colagrossi, D. Le Touzé, and B. Alessandrini, "An hamiltonian interface sph formulation for multi-fluid and free surface flows," Journal of Computational Physics, vol. 228, no. 22, pp. 8380-8393, 2009.

[19] S. J. Neethling and D. J. Barker, "Using smooth particle hydrodynamics (sph) to model multiphase mineral processing systems," Minerals Engineering, vol. 90, pp. 17-28, 2016.

[20] S. J. Neethling and D. J. Barker, "Using smooth particle hydrodynamics (sph) to model multiphase mineral processing systems," Minerals Engineering, vol. 90, pp. 17-28, 2016.

[21] N. Grenier, Modélisation numérique par la méthode SPH de la séparation eau-huile dans les séparateurs gravitaires. PhD thesis, Ecole Centrale de Nantes (ECN)(ECN)(ECN)(ECN), 2009.

[22] J. Hunter, Surface tension in smoothed particle hydrodynamics. PhD thesis, Honours thesis, Mathematics Department, Monash University, Melbourne, Australia, 1992.

[23] A. Tartakovsky and P. Meakin, "Modeling of surface tension and contact angles with smoothed particle hydrodynamics," Physical Review E, vol. 72, no. 2, p. 026301, 2005.

[24] A. M. Tartakovsky and P. Meakin, "A smoothed particle hydrodynamics model for miscible flow in three-dimensional fractures and the two-dimensional rayleigh-taylor instability," Journal of Computational Physics, vol. 207, no. 2, pp. 610-624, 2005.

[25] J. Li, "An arbitrary lagrangian eulerian method for three-phase flows with triple junction points," Journal of Computational Physics, vol. 251, pp. 1-16, 2013.

[26] J. J. Monaghan, "Simulating free surface flows with sph," Journal of computational physics, vol. 110, no. 2, pp. 399-406, 1994. 
[27] R. Sivanesapillai, N. Falkner, A. Hartmaier, and H. Steeb, "A csf-sph method for simulating drainage and imbibition at pore-scale resolution while tracking interfacial areas," Advances in Water Resources, vol. 95, pp. 212-234, 2016.

[28] A. Colagrossi and M. Landrini, "Numerical simulation of interfacial flows by smoothed particle hydrodynamics," Journal of Computational Physics, vol. 191, no. 2, pp. 448-475, 2003.

[29] S. Marrone, A. Colagrossi, M. Antuono, G. Colicchio, and G. Graziani, "An accurate sph modeling of viscous flows around bodies at low and moderate reynolds numbers," Journal of Computational Physics, vol. 245, pp. 456-475, 2013.

[30] M. Liu, G. Liu, and K. Lam, "Constructing smoothing functions in smoothed particle hydrodynamics with applications," Journal of Computational and applied Mathematics, vol. 155, no. 2, pp. 263-284, 2003.

[31] J. P. Morris, Analysis of smoothed particle hydrodynamics with applications. Monash University Australia, 1996.

[32] X. Hu and N. A. Adams, "An incompressible multi-phase sph method," Journal of computational physics, vol. 227, no. 1, pp. 264-278, 2007.

[33] X. Hu and N. Adams, "Angular-momentum conservative smoothed particle dynamics for incompressible viscous flows," Physics of Fluids, vol. 18, no. 10, p. 101702, 2006.

[34] J. Monaghan and R. Gingold, "Shock simulation by the particle method sph," Journal of computational physics, vol. 52, no. 2, pp. 374-389, 1983.

[35] J. J. Monaghan, "Smoothed particle hydrodynamics," Reports on progress in physics, vol. 68, no. 8, p. 1703, 2005.

[36] D. J. Price, "Smoothed particle magnetohydrodynamics-iv. using the vector potential," Monthly Notices of the Royal Astronomical Society, vol. 401, no. 3, pp. 1475-1499, 2010.

[37] S. Adami, X. Hu, and N. Adams, "Contact line hydrodynamics with sph," in 7 th international SPHERIC workshop, 2012. 
[38] J. J. Monaghan and J. B. Kajtar, "Sph particle boundary forces for arbitrary boundaries," Computer Physics Communications, vol. 180, no. 10, pp. 1811-1820, 2009.

[39] R. Gesztelyi, J. Zsuga, A. Kemeny-Beke, B. Varga, B. Juhasz, and A. Tosaki, "The hill equation and the origin of quantitative pharmacology," Archive for history of exact sciences, vol. 66, no. 4, pp. 427-438, 2012.

[40] F. Boyer, C. Lapuerta, S. Minjeaud, B. Piar, and M. Quintard, "Cahnhilliard/navier-stokes model for the simulation of three-phase flows," Transport in Porous Media, vol. 82, no. 3, pp. 463-483, 2010.

[41] J. Monaghan and A. Rafiee, "A simple sph algorithm for multi-fluid flow with high density ratios," International Journal for Numerical Methods in Fluids, vol. 71, no. 5, pp. 537-561, 2013.

[42] D. Layzer, "On the instability of superposed fluids in a gravitational field.," The astrophysical journal, vol. 122, p. 1, 1955.

[43] S.-R. Hysing, S. Turek, D. Kuzmin, N. Parolini, E. Burman, S. Ganesan, and L. Tobiska, "Quantitative benchmark computations of twodimensional bubble dynamics," International Journal for Numerical Methods in Fluids, vol. 60, no. 11, pp. 1259-1288, 2009.

[44] M. Sussman, P. Smereka, and S. Osher, "A level set approach for computing solutions to incompressible two-phase flow," Journal of Computational physics, vol. 114, no. 1, pp. 146-159, 1994.

[45] D. Shepard, "A two-dimensional interpolation function for irregularlyspaced data," in Proceedings of the 1968 23rd ACM National Conference, ACM '68, (New York, NY, USA), pp. 517-524, ACM, 1968.

[46] S. Popinet and S. Zaleski, "A front-tracking algorithm for accurate representation of surface tension," International Journal for Numerical Methods in Fluids, vol. 30, no. 6, pp. 775-793, 1999.

[47] D. Torres and J. Brackbill, "The point-set method: front-tracking without connectivity," Journal of Computational Physics, vol. 165, no. 2, pp. 620-644, 2000 . 
[48] D. Jamet, D. Torres, and J. Brackbill, "On the theory and computation of surface tension: the elimination of parasitic currents through energy conservation in the second-gradient method," Journal of Computational Physics, vol. 182, no. 1, pp. 262-276, 2002.

[49] J. Kim, "A diffuse-interface model for axisymmetric immiscible twophase flow," Applied mathematics and computation, vol. 160, no. 2, pp. 589-606, 2005.

[50] J. S. Rowlinson and B. Widom, Molecular theory of capillarity. Courier Corporation, 2013.

[51] H. G. Lee, K. Kim, and J. Kim, "On the long time simulation of the rayleigh-taylor instability," International Journal for Numerical Methods in Engineering, vol. 85, no. 13, pp. 1633-1647, 2011.

[52] S. J. Cummins and M. Rudman, "An sph projection method," Journal of computational physics, vol. 152, no. 2, pp. 584-607, 1999.

[53] H. Wendland, "Piecewise polynomial, positive definite and compactly supported radial functions of minimal degree," Advances in computational Mathematics, vol. 4, no. 1, pp. 389-396, 1995.

[54] S. Dalziel, "Toy models for rayleigh taylor instability," in 8th International Workshop on the Physics of Compressible Turbulent Mixing, 2001.

[55] G. A. Greene, J. C. Chen, and M. T. Conlin, "Onset of entrainment between immiscible liquid layers due to rising gas bubbles," International Journal of Heat and Mass Transfer, vol. 31, no. 6, pp. 1309-1317, 1988.

[56] G. A. Greene, J. C. Chen, and M. T. Conlin, "Bubble induced entrainment between stratified liquid layers," International journal of heat and mass transfer, vol. 34, no. 1, pp. 149-157, 1991.

[57] N. Grenier, D. Le Touzé, A. Colagrossi, M. Antuono, and G. Colicchio, "Viscous bubbly flows simulation with an interface sph model," Ocean Engineering, vol. 69, pp. 88-102, 2013. 


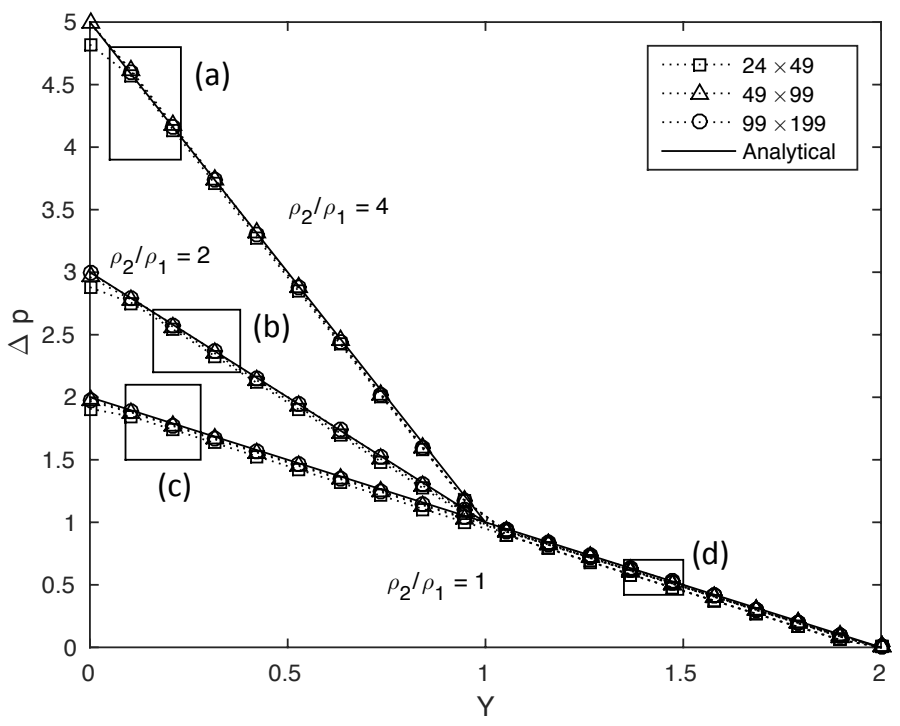

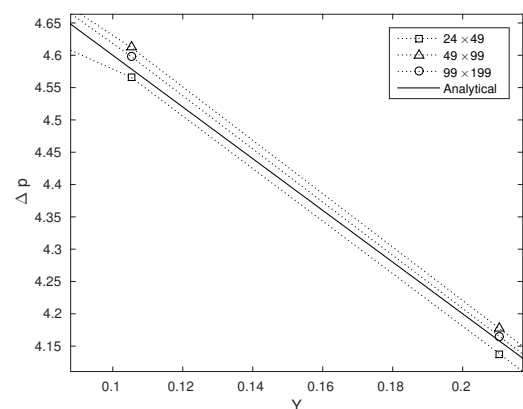

(a)

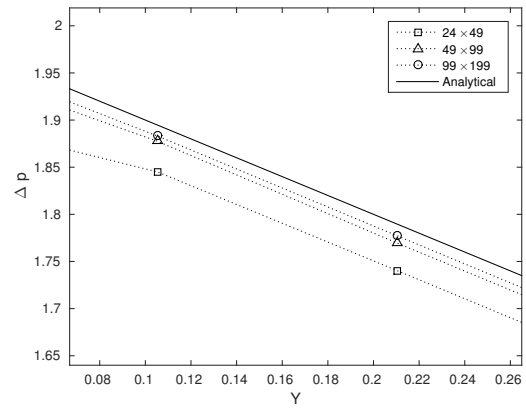

(c)

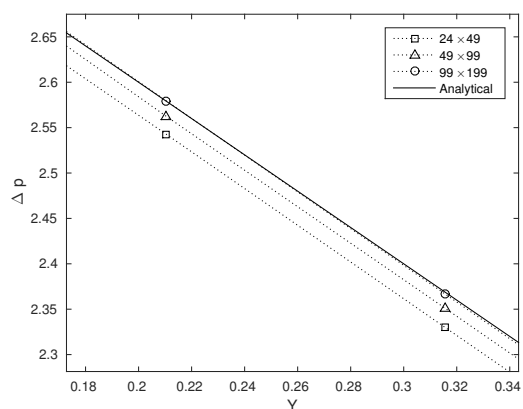

(b)

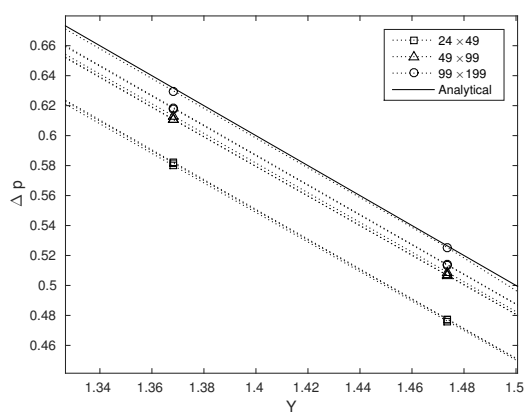

(d)

Figure 20: Hydrostatic pressure variation $\Delta p=p-p_{\min }$ for the three different density ratios $\frac{\rho_{2}}{\rho_{1}}$. Below, we plot a zoom of the selected zones $(a)(b)(c)(d)$. 


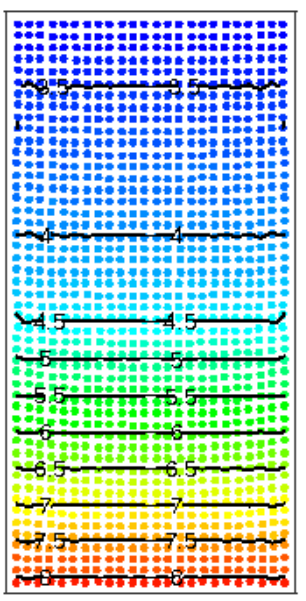

$24 \times 49$

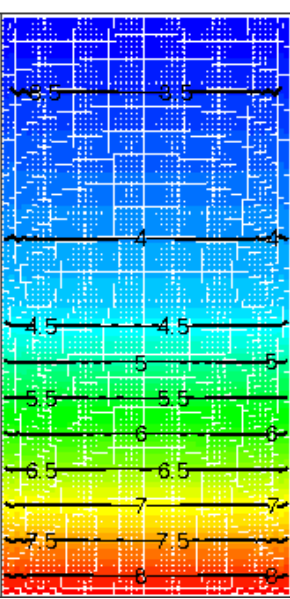

$49 \times 99$

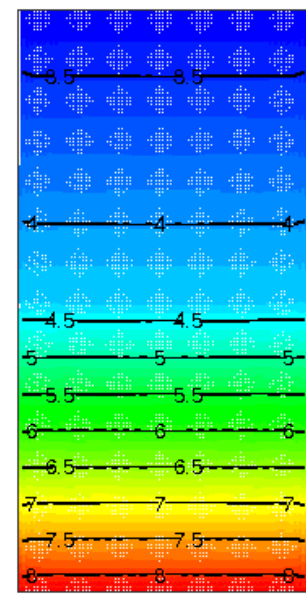

$99 \times 199$

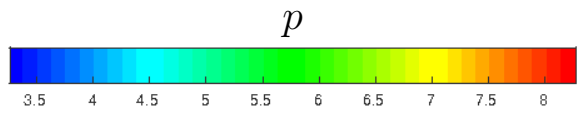

Figure 21: The fluid column particles colored with normalized hydrostatic pressure for the density ratio $\frac{\rho_{2}}{\rho_{1}}=4$ with the three particle resolutions $24 \times 49$ (left), $49 \times 99$ (center), $99 \times 199$ (right). It is also shown the pressure isolines in the range 3.5 to $8[\mathrm{~Pa}]$.

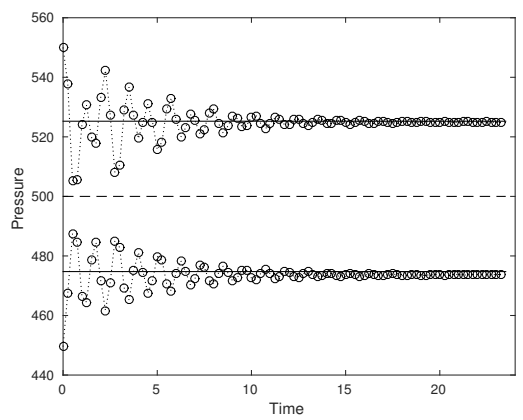

Figure 22: Time-evolution of pressure at the point $(0.5,0.5)$ (upper curves) and $(0.5,1.5)$ (lower curves) after the damping period for the for the density and viscosity ratios of $\frac{\rho_{2}}{\rho_{1}}=100$ and $\frac{\mu_{2}}{\mu_{1}}=10$. The solid lines represent the stabilized pressure, and the dashed line presents the background pressure. 


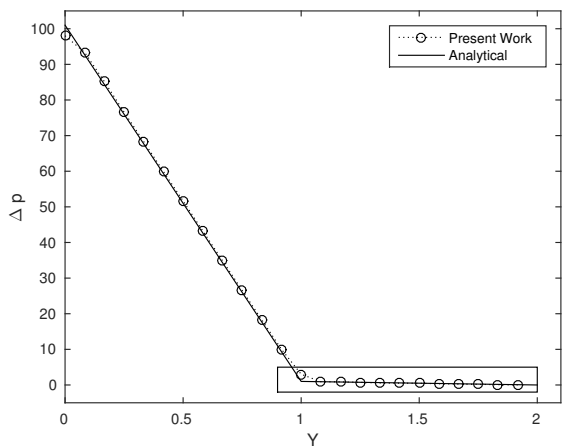

$(a)$

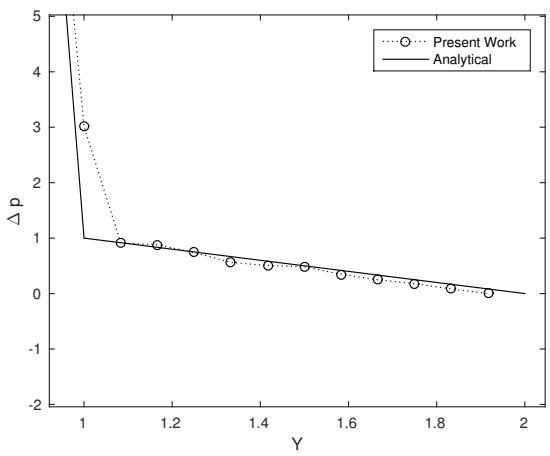

(b)

Figure 23: Hydrostatic pressure variation $\Delta p=p-p_{\text {min }}$ for the density and viscosity ratios of $\frac{\rho_{2}}{\rho_{1}}=100$ and $\frac{\mu_{2}}{\mu_{1}}=10$. On the right we plot a zoom of the selected zone.
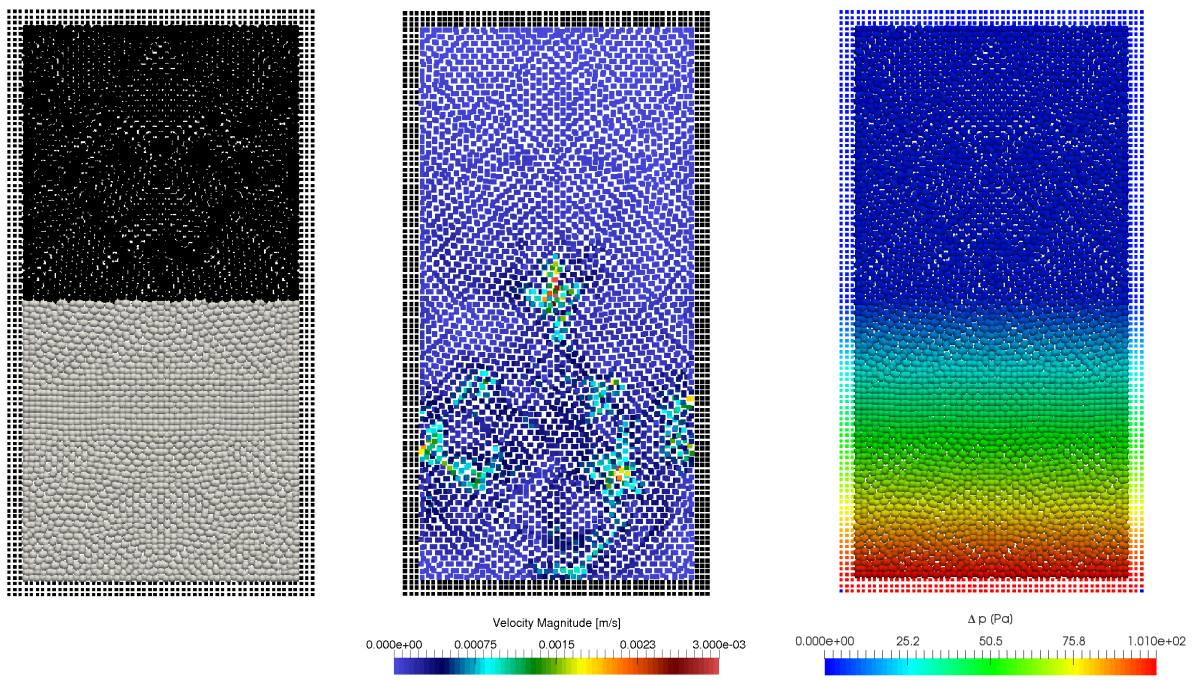

Figure 24: The fluid column particles for the density and viscosity ratios of $\frac{\rho_{2}}{\rho_{1}}=100$ and $\frac{\mu_{2}}{\mu_{1}}=10$. The left figure represents the fluid particles colored with phase color (gray color for fluid 2, black color for fluid 1). The figure of the center show the velocity magnitude holds on every fluid particle. The right figure represents the fluid and wall particles colored with hydrostatic pressure filed. 


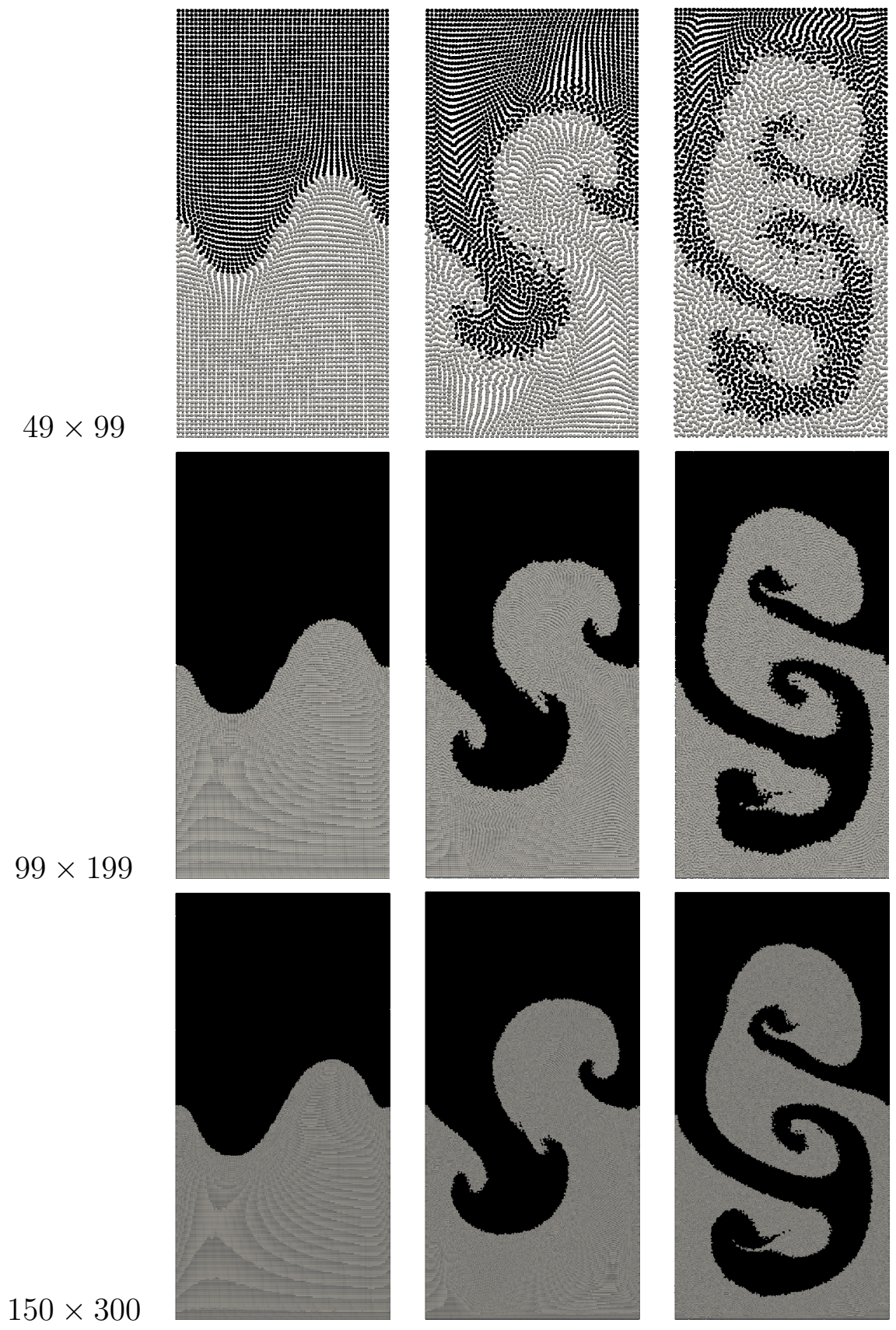

Figure 25: Rayleigh-Taylor instability at three different times $t=1[s]$ (left column), $t=3[s]$ (center column), $t=5[s]$ (right column) after the damping period, and three different resolutions: $49 \times 99$ particles (top), $99 \times 199$ particles (middle) and $150 \times 300$ particles (bottom). 

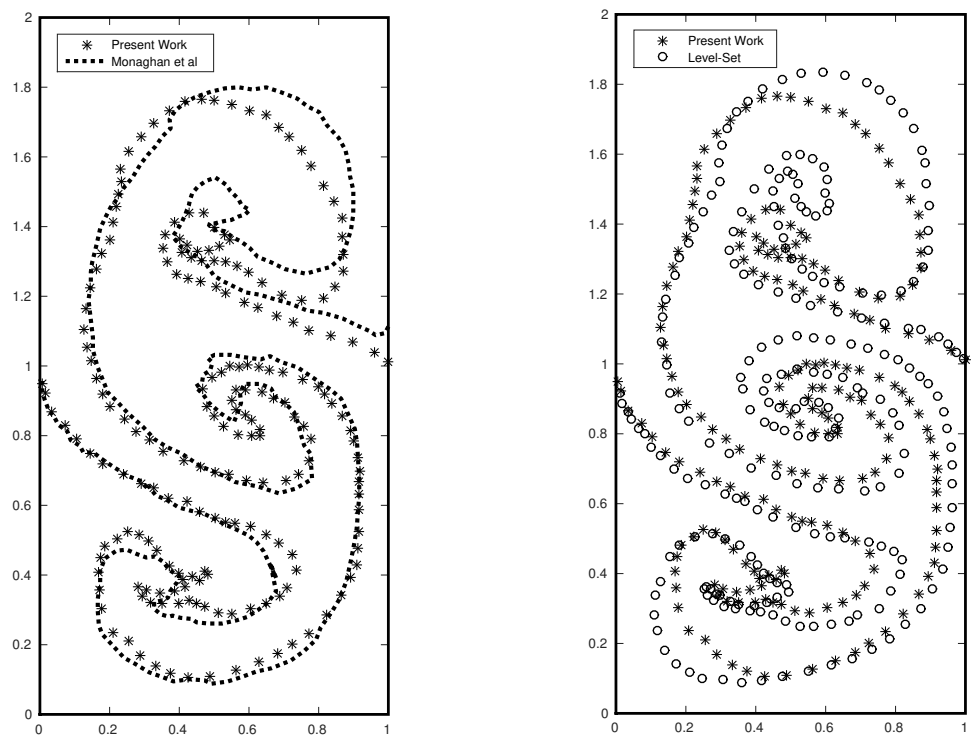

Figure 26: Comparison between the fluid interfaces of the present work and the references. The left figure compares the fluid interface of present SPH model with that of SPH model developed by Monaghan et al [41].The right figure compares the fluid interface of the present SPH method with that of Level-Set method [18].

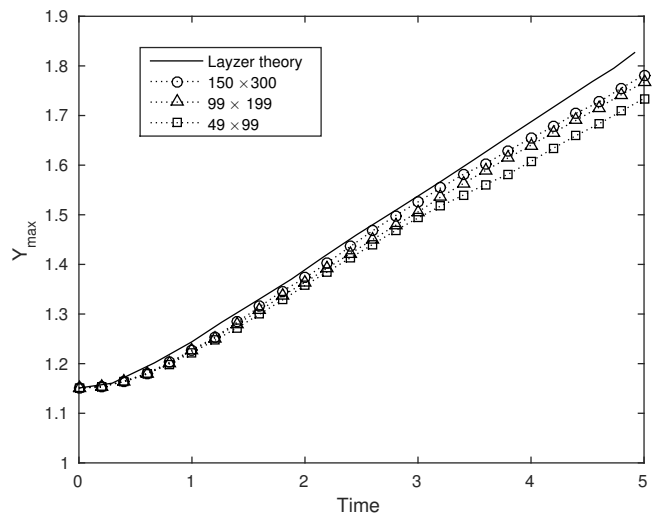

Figure 27: Time-evolution for the highest point $\left(Y_{\max }\right)$ of the lighter fluid. Dotted line with small open triangles shows the results for the simulation with $99 \times 199$ particles, the small open square for $49 \times 99$ particles and the small open circles for $150 \times 300$ particles. Layzer's theory curve is represented by the continuous solid line. 

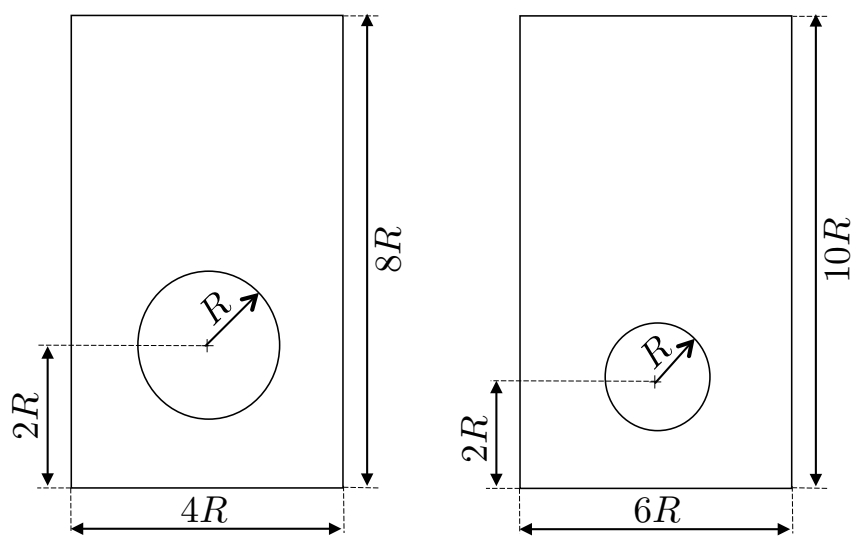

Figure 28: Single bubble rising through a vertical column of water. Geometrical details of the initial setup. On the left it is schematically described the low-density ratio configuration (Case 1)[43] $(R=0.25[\mathrm{~m}])$ and on the right it is described the setup for the high-density ratio configuration (Case 2) $[44](R=0.025[\mathrm{~m}])$.
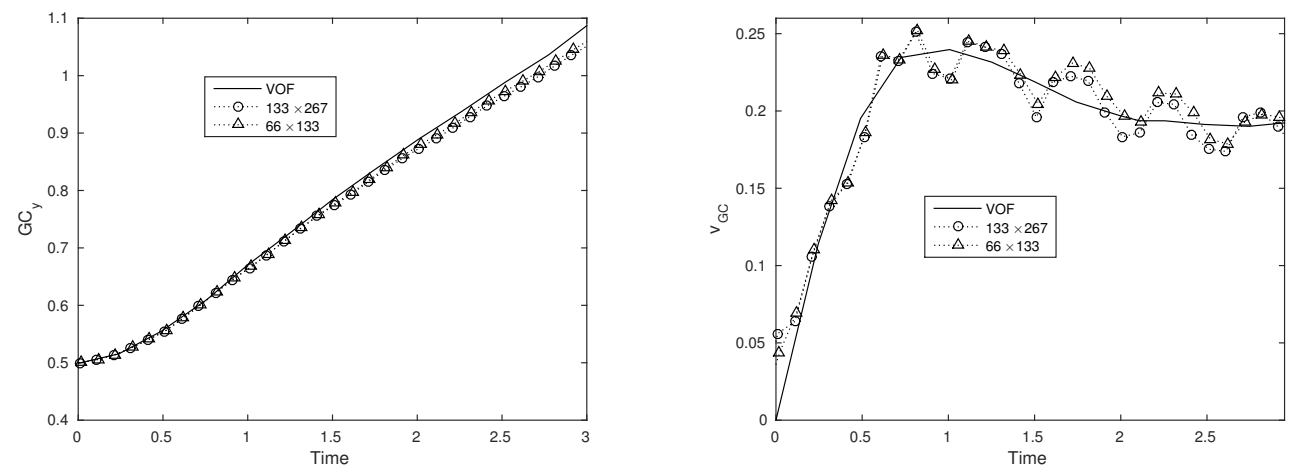

Figure 29: Single bubble rising through a vertical column of water. Case 1: Time evolution of the position of the gravity center (left) and vertical velocity (right) of the rising bubble for different number of particles. The solid line represents the results obtained by VOF method [43]. 


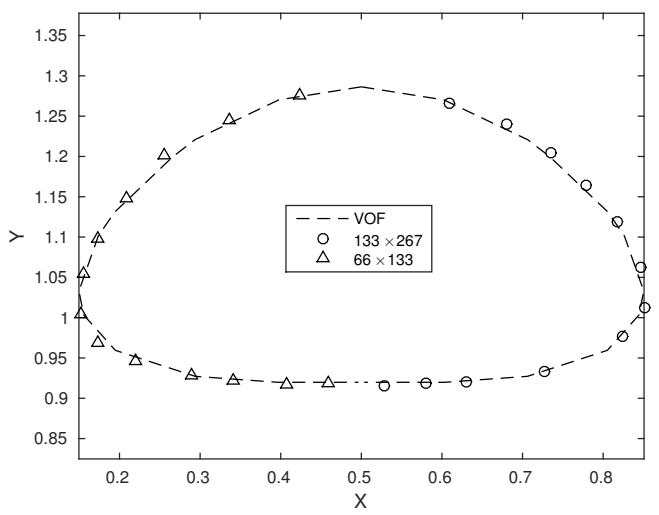

Figure 30: Single bubble rising through a vertical column of water. Case 1: Comparison between the bubble interfaces at time $t=3[s]: 66 \times 133 \mathrm{SPH}$ (Small triangles), $133 \times 267$ SPH (Small circles). Dashed line represent the interface obtained with the VOF method [43]. 

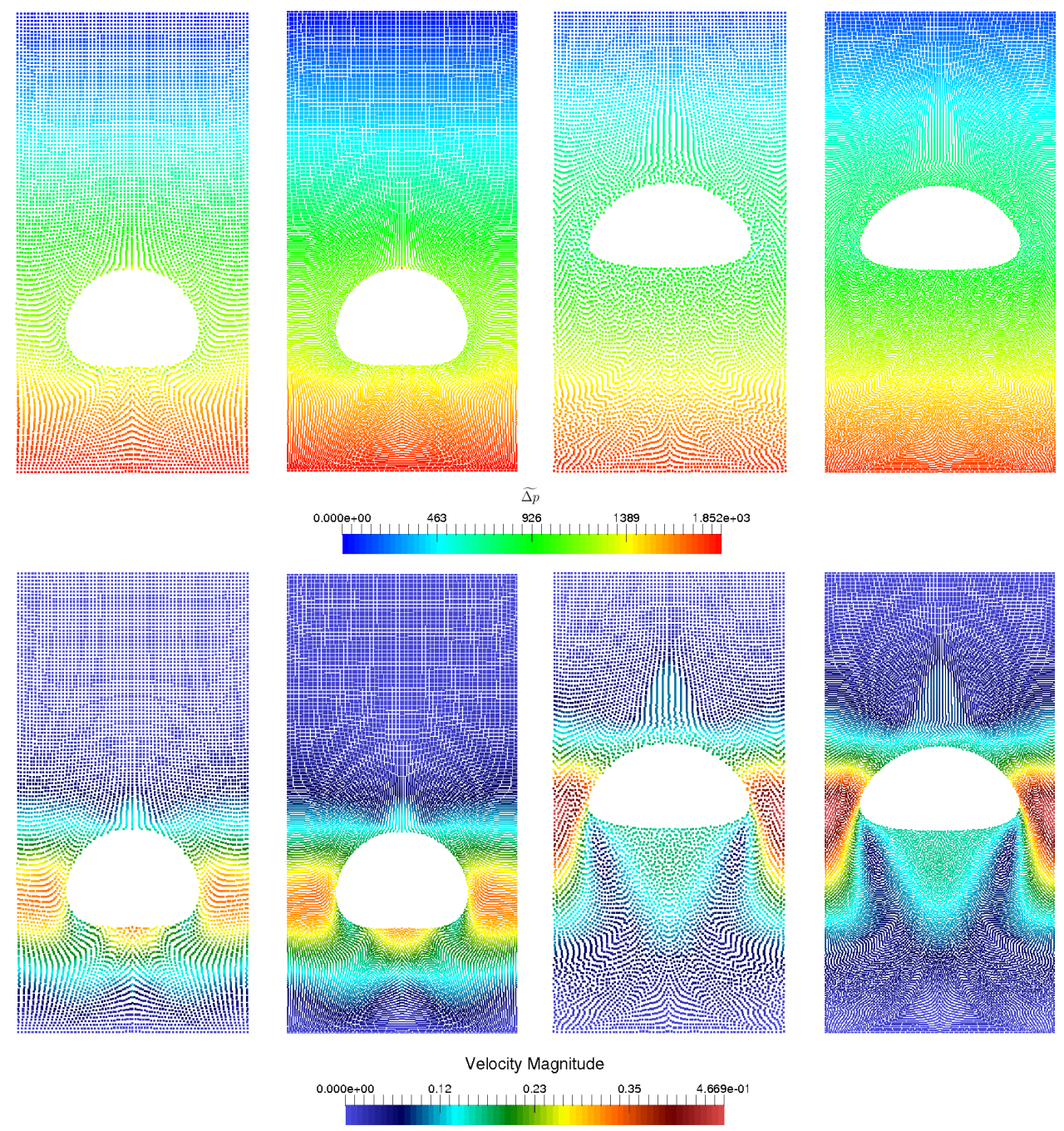

$$
\mathrm{t}=1[\mathrm{~s}] \quad \mathrm{t}=3[\mathrm{~s}]
$$

Figure 31: Single bubble rising through a vertical column of water. Case 1: Pressure (top) and velocity (bottom) fields at times $t=1[s]$ and $t=3[s]$ for two different particle resolutions, $66 \times 133$ (left) and $133 \times 267$ (right). 


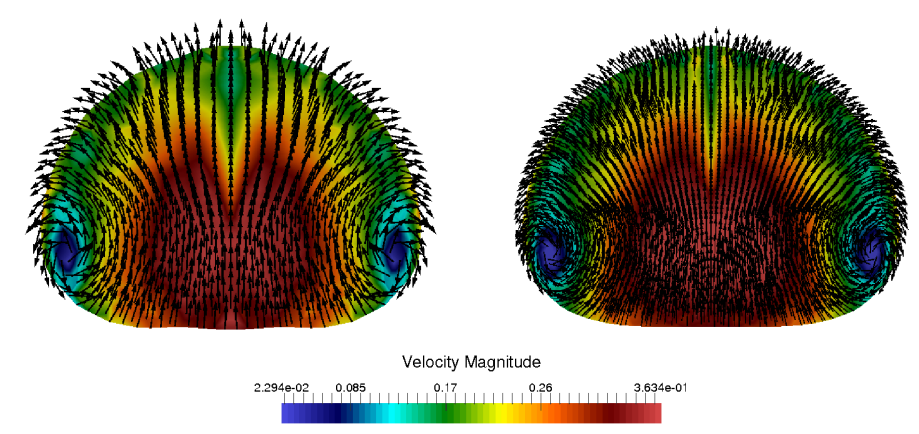

Figure 32: Single bubble rising through a vertical column of water. Case 1: Direction of the velocity vector of the particles inside the bubble at $t=1$ colored with magnitude velocity. On the left, results for $66 \times 133$ particles, and on the right we plot the results for the $133 \times 267$ particles case.
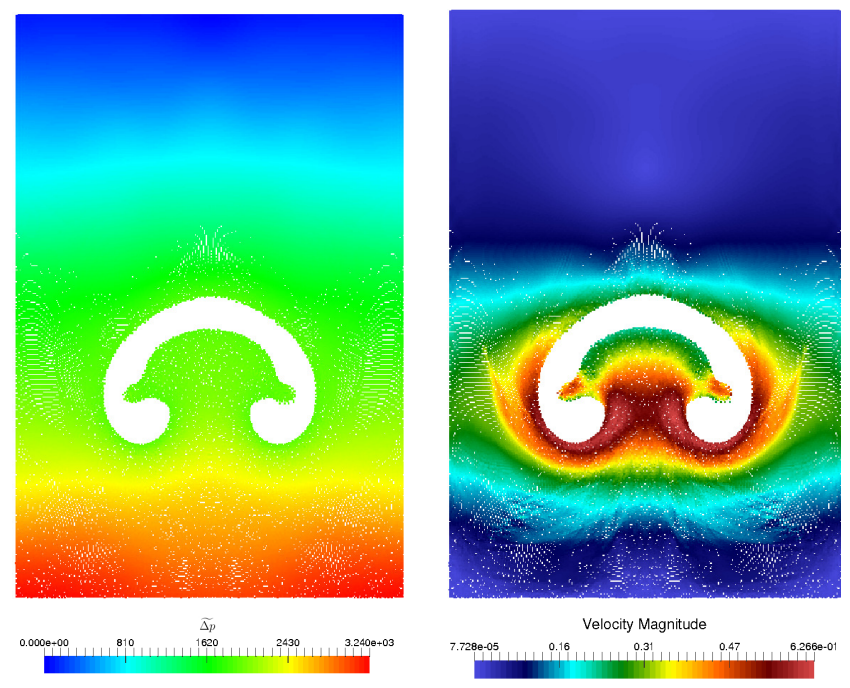

Figure 33: Single bubble rising through a vertical column of water. Case 2: Relative pressure $\left(\widetilde{\Delta p}=p-p_{\min }\right)$ and magnitude of the velocity of the column of water at the dimensionless time $t \sqrt{\|\mathbf{g}\| / R}=3.6$ 

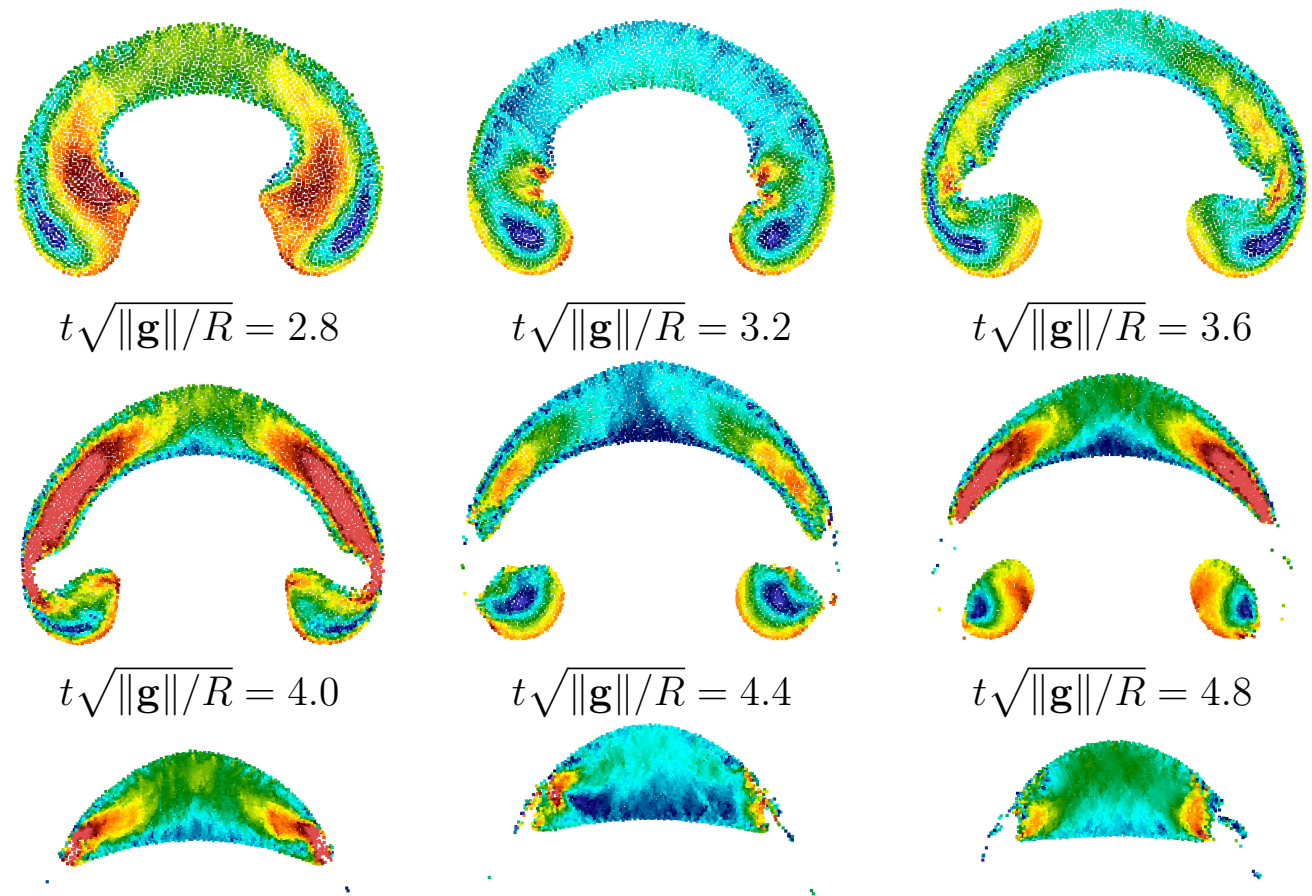

$t \sqrt{\|\mathbf{g}\| / R}=4.8$
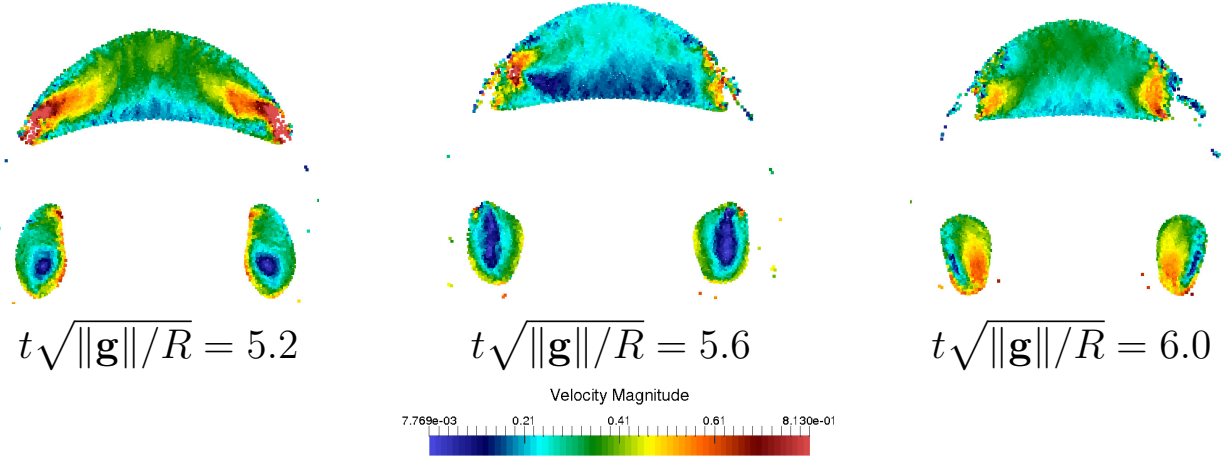

$t \sqrt{\|\mathbf{g}\| / R}=6.0$

Figure 34: Single bubble rising through a vertical column of water. Case 2: Evolution of the bubble at nine different instants. The Bubble is colored with velocity magnitude field. 

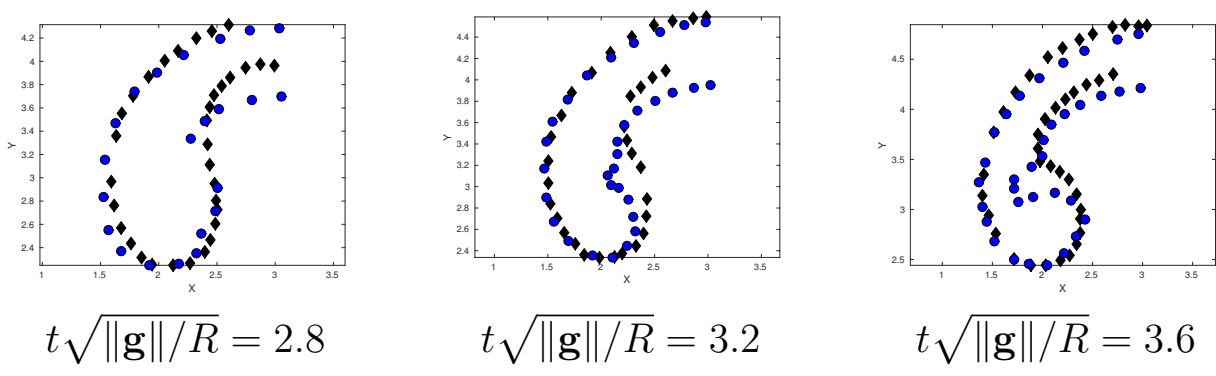

$$
t \sqrt{\|\mathbf{g}\| / R}=3.6
$$
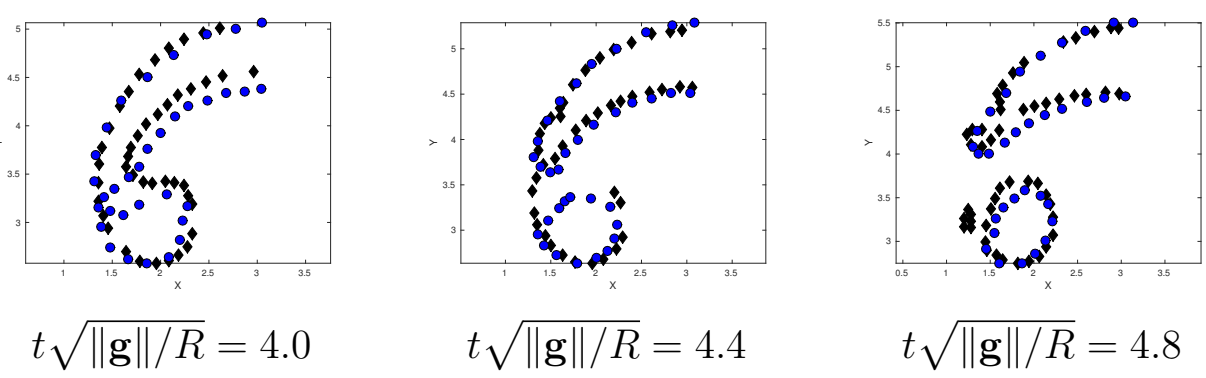

$$
t \sqrt{\|\mathbf{g}\| / R}=4.4
$$
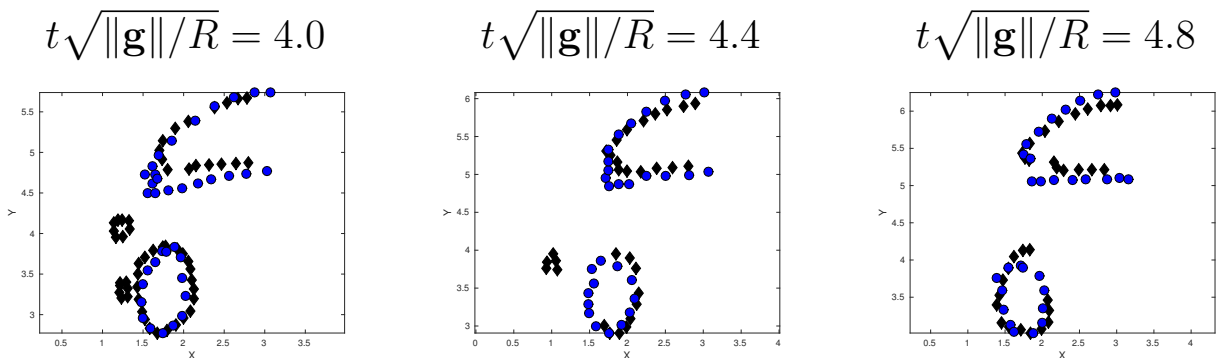

$$
t \sqrt{\|\mathbf{g}\| / R}=5.2
$$

$$
t \sqrt{\|\mathbf{g}\| / R}=6.0
$$

Figure 35: Single bubble rising through a vertical column of water. Case 2: Evolution of the bubble at nine different instants. Results obtained with the present SPH method (blue circles) and with a Level-Set approach [44] (black diamonds). 


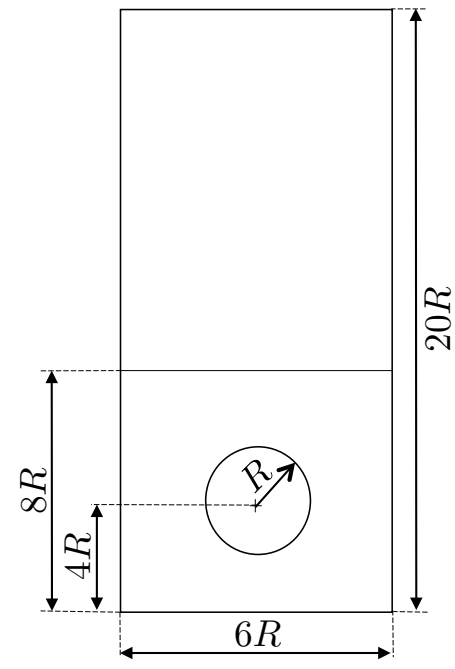

Figure 36: Gas bubble rising through two stratified fluid layers. Geometrical details of the problem setup. 

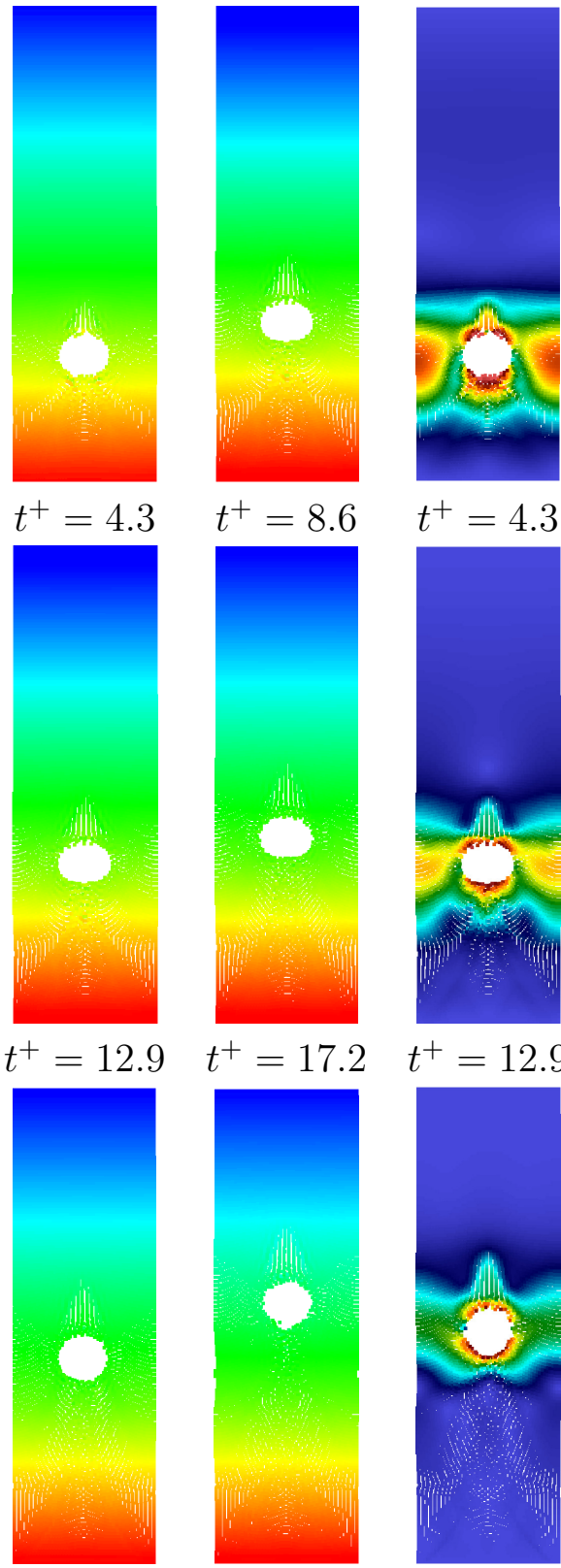

$t^{+}=21.5$
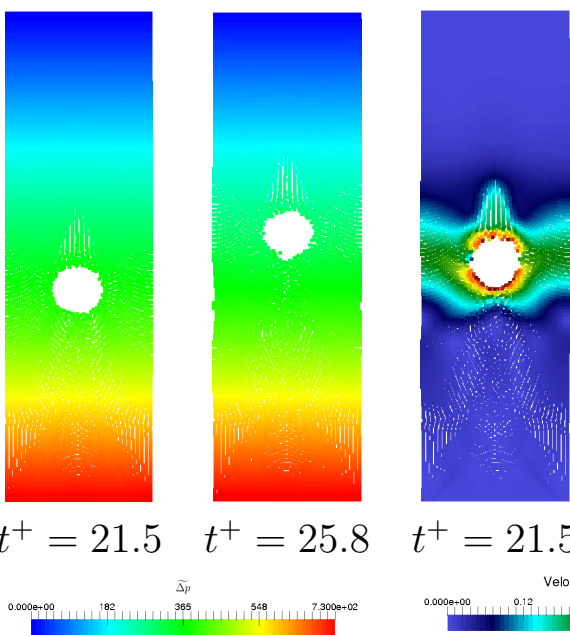
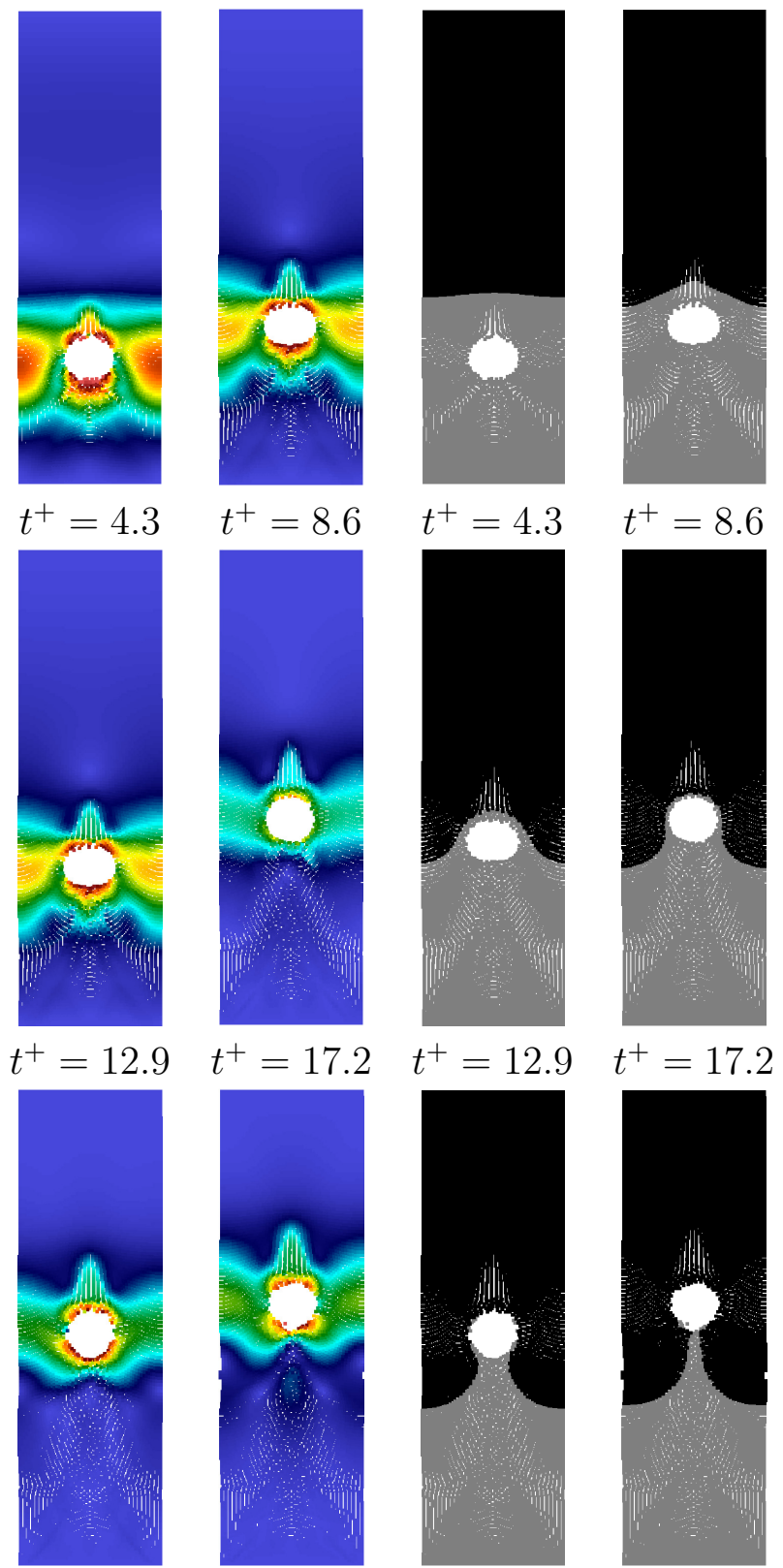

$t^{+}=21.5$

$t^{+}=25.8$

$t^{+}=21.5$

$t^{+}=25.8$

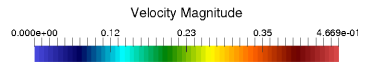

fluid 2

fluid 3

Figure 37: Gas bubble rising through two stratified fluid layers. Starting from the left, first and second columns show the relative hydrostatic pressure field $\widetilde{\Delta p}=p-p_{\text {min }}$ for different normalized times $t^{+}=t \sqrt{\|\mathbf{g}\| / R}$. Third and fourth columns present the magnitude of the velocity field. The last two columns show the fluid phases distribution. 


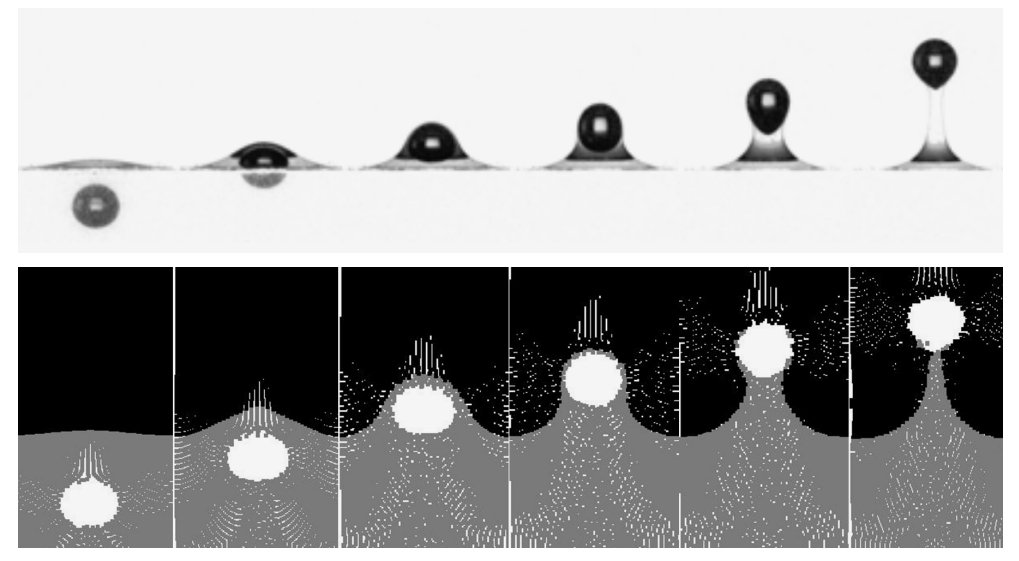

Figure 38: Gas bubble rising through two stratified fluid layers. On the top, we show the experimental sequence taken from [4]. On the bottom we plot the results obtained using the proposed SPH method. The dimensionless interval between two images is taken equal to $\Delta t \sqrt{\|\mathbf{g}\| / R}=4.3$

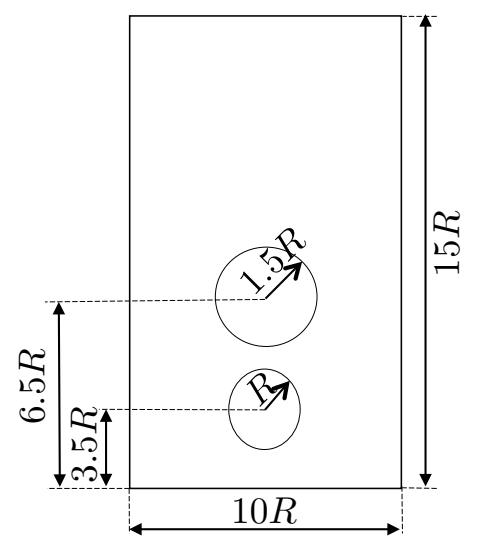

Figure 39: Interaction of two rising bubbles through a fluid column: Geometrical setup. 

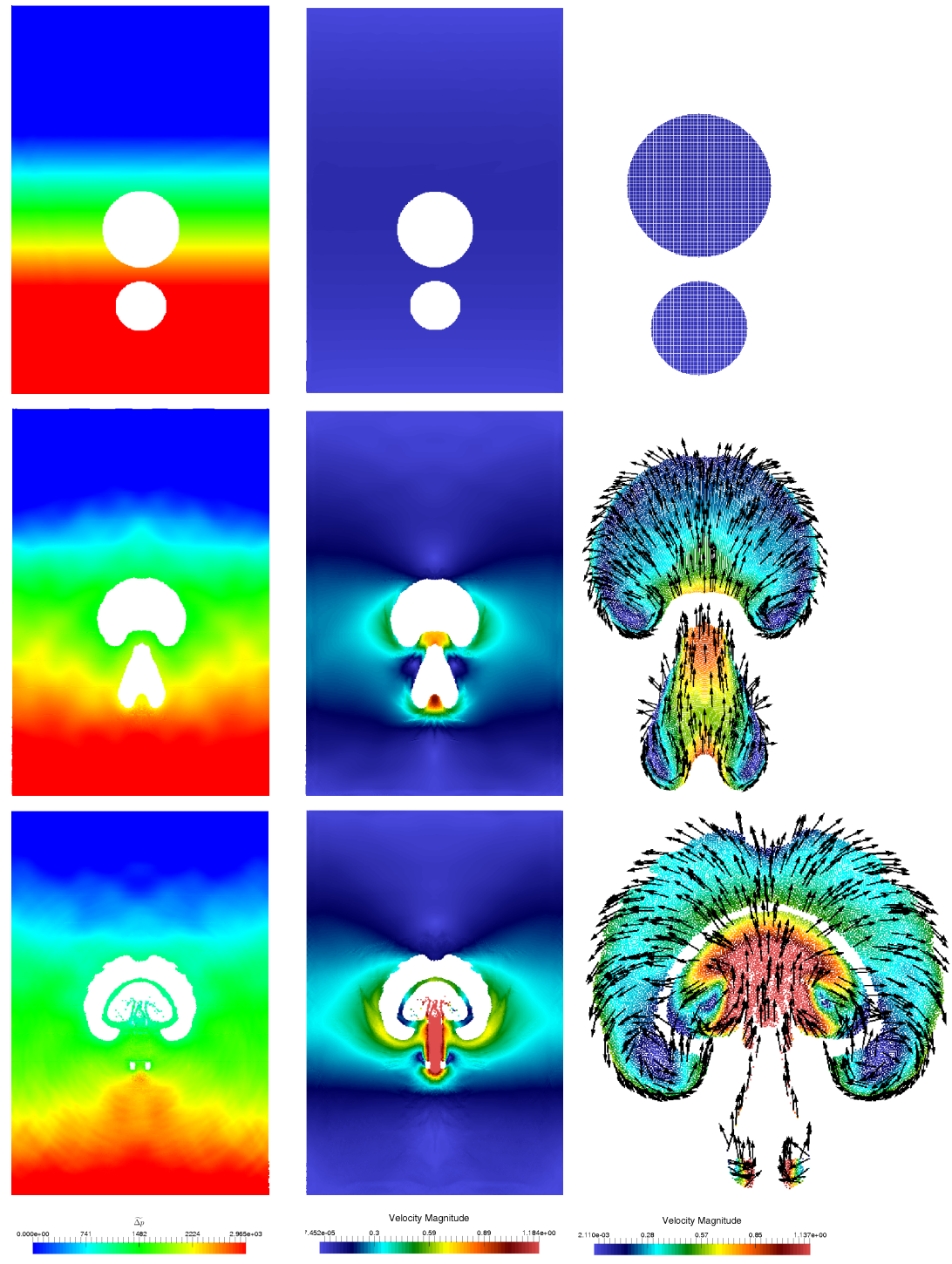

Figure 40: Interaction of two rising bubbles through a fluid column First and second images columns represent the pressure and magnitude velocity, respectively. The third column presents the magnitude velocity with particle direction vector of isolated bubble. The simulations are presented in order at the times $t=\{0,0.25,0.5\}[s]$. 

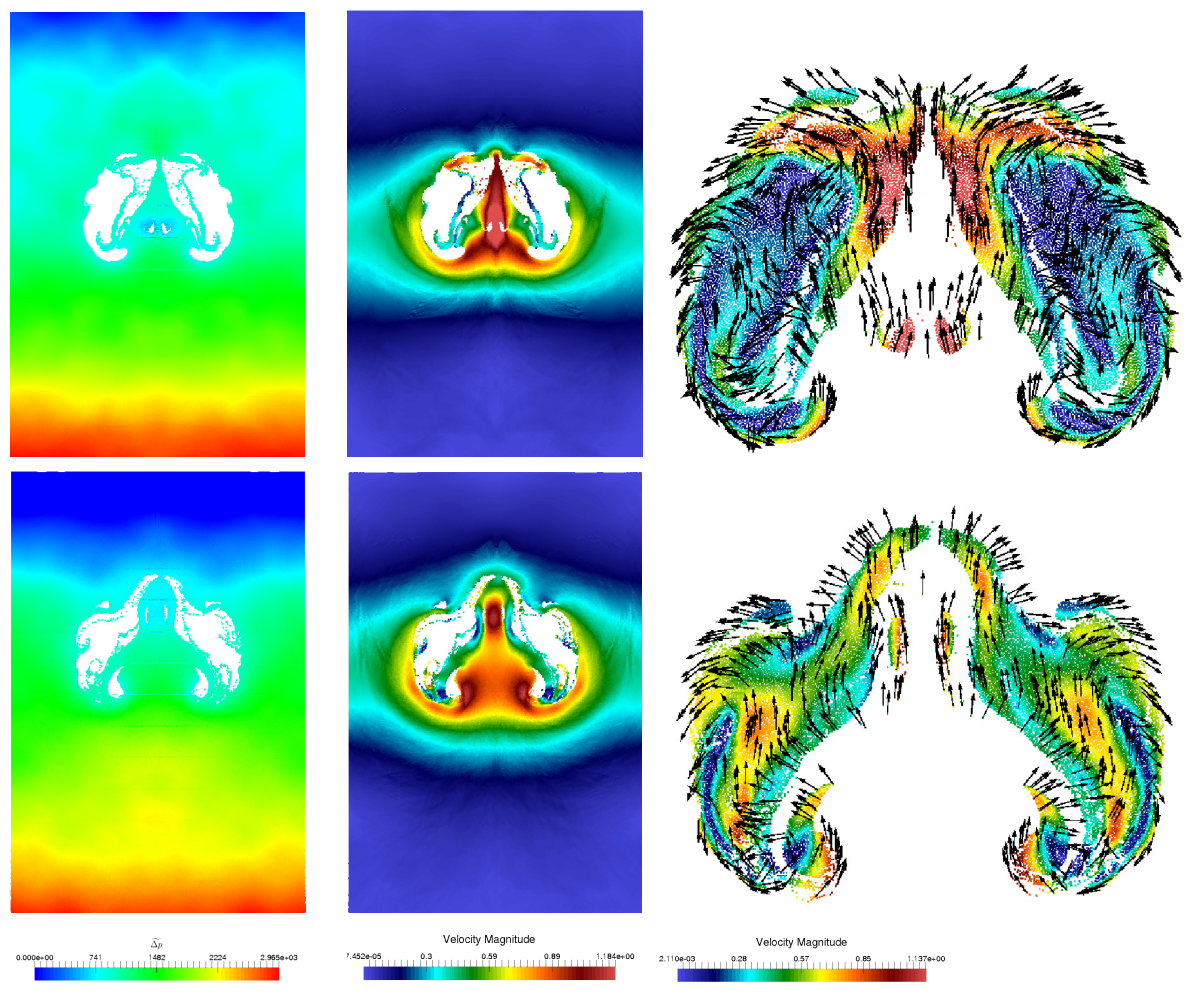

Figure 41: Two rising bubbles through a fluid column. First and second images columns represent the pressure and magnitude velocity, respectively. The third column presents the magnitude velocity with particle direction vector of isolated bubble. The simulations are presented in order at the times $t=\{0.75,1\}[s]$.

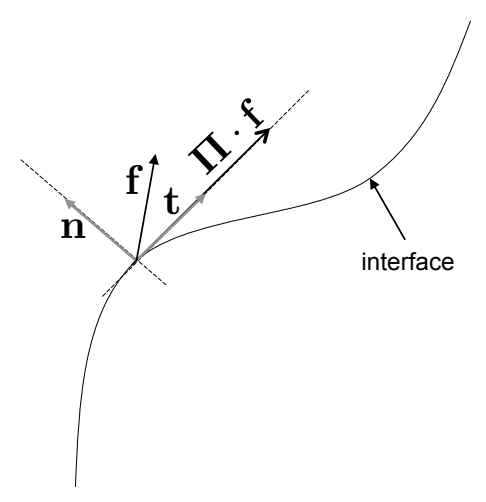

Figure 42: Application of stress surface tensor $\boldsymbol{\Pi}$ on an arbitrary vector $\mathbf{f}$. Where $\mathbf{n}$ and $\mathbf{t}$ are the normal and tangential vectors on the interface, respectively. 
The physical proprieties of the heavier phase must be assigned to all fluid phases;

The initial particles velocity and pressure are set to zero;

Set $c_{0}, p_{b}, \delta t, T_{D}, T_{e n d}$

while $\delta t * i_{t}<T_{\text {end }}$ do

Search for each particle $i \in \Omega=\Omega_{f} \cup \Omega_{s}$ its particles neighbor $j$. /* linked list method is employed in this work. $\Omega_{f}$ and $\Omega_{s}$ denote the fluid and solid particles, respectively.

/* Damping process

if $\delta t * i_{t}<T_{D}$ then

I Compute $\xi_{D}$ using Eq.37;

end

if $i_{t}=\left\lceil\frac{T_{D}}{\delta t}\right\rceil$ then

I Assign the real physical proprieties to each fluid phase;

end

for $i \in \Omega_{s}$ do

| Compute $p_{w}$ and $\mathbf{v}_{\mathbf{w}}$ using Eqs.25 and 23, respectively; end

/* Prediction Step

for $i \in \Omega_{f}$ do

Compute $\nabla p_{i}, \mathbf{F}_{\mathbf{i}}{ }^{V i s}$ and $\mathbf{F}_{\mathbf{i}}{ }^{S T}$ using Eqs.26, 28 and 22, respectively;

$\widetilde{\mathbf{v}}_{\mathbf{i}}^{n+1}=\mathbf{v}_{\mathbf{i}}^{n}+\delta t\left(\frac{1}{\rho_{i}}\left(-\nabla p_{i}+\mathbf{F}_{\mathbf{i}}{ }^{V i s}+\mathbf{F}_{\mathbf{i}}{ }^{S T}\right)^{n}+\xi_{D} \mathbf{g}\right)$

$\widetilde{\mathbf{r}}_{\mathbf{i}}^{n+1}=\mathbf{r}_{\mathbf{i}}^{n}+\delta t \mathbf{v}_{i}^{n}$

end

for $i \in \Omega$ do

/* In this loop, the predicted particle velocities and positions are used

for the calculation of $\Pi_{i}, p_{w}$ and $\mathbf{v}_{\mathbf{w}}$.

if $i \in \Omega_{f}$ and $\delta t * i_{t} \geq T_{D}$ then

Compute $\Pi_{i}$ using Eq.18;

end

if $i \in \Omega_{s}$ then

end

Compute $p_{w}, \mathbf{v}_{\mathbf{w}}$ using Eqs 25 and 23, respectively;

end

/* Correction Step

for $i \in \Omega_{f}$ do

/* In this loop, the predicted particle velocities and positions are used

for the calculation of $\nabla p_{i}, \mathbf{F}_{\mathbf{i}}{ }^{V i s}$ and $\mathbf{F}_{\mathbf{i}} S T$

Compute $\nabla p_{i}, \mathbf{F}_{\mathbf{i}}{ }^{V i s}$ and $\mathbf{F}_{\mathbf{i}}{ }^{S T}$ using Eqs.26, 28 and 22, respectively;

$\mathbf{v}_{\mathbf{i}}{ }^{n+1}=\frac{1}{2}\left\{\mathbf{v}_{\mathbf{i}}{ }^{n}+\widetilde{\mathbf{v}}_{\mathbf{i}}^{n+1}\right\}+\frac{\delta t}{2}\left(\frac{1}{\rho_{i}}\left(-\nabla p_{i}+\mathbf{F}_{\mathbf{i}}{ }^{V i s}+\mathbf{F}_{\mathbf{i}}{ }^{S T}\right)^{n}+\xi_{D} \mathbf{g}\right) ;$

$\mathbf{r}_{\mathbf{i}}^{n+1}=\mathbf{r}_{\mathbf{i}}^{n}+\delta t \mathbf{v}_{i}^{n+1}$

end

for $i \in \Omega_{f}$ do

/* In this loop, the predicted particle positions are used for the calculation of $\rho_{i}$ and thus $p_{i}$. 